AQUATIC MACROPHYTES AND SELECTED PHYSICAL PROPERTIES OF SHINGOBEE AND WILLIAMS LAKES, MINNESOTA, 1991-92

by Virginia Carter, P.T. Gammon, D.O. Rosenberry, and Michael Turtora

U. S. GEOLOGICAL SURVEY

Open-File Report 93-143

Reston, Virginia

1993 


\section{U. S. DEPARTMENT OF THE INTERIOR}

BRUCE BABBITT, Secretary

U. S. GEOLOGICAL SURVEY

Dallas L. Peck, Director

For additional information write to:

Chief, Branch of Regional Research Northeastern Region U.S. Geological Survey 430 National Center 12201 Sunrise Valley Drive Reston, Virginia 22092
Copies of this report can be purchased from:

U.S. Geological Survey Books and Open-File Reports Box 25286, Federal Center Denver, Colorado 80225 


\section{CONTENTS}

Abstract
Introduction
Acknowledgments
Description of Study area
Collection and Analysis of Aquatic Macrophyte and Physical Property Data
References f. Aquatic macrophyte species and biomass data
Appendix 1. Plant nutrient composition
3. Plant Species in the Emergent Zones of Shingobee and Williams Lakes
4. Lake hypsographic data
5. Light attenuation data

\section{ILLUSTRATIONS}

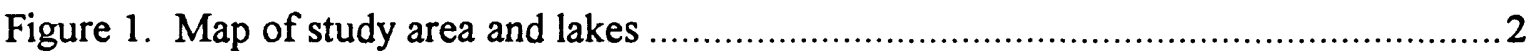

2. Bathymetric map of Shingobee Lake showing location of transects...................... 4

3. Bathymetric map of Williams Lake showing location of transects .....................5

4. Graph showing depth-volume curves for Williams and Shingobee Lakes.............7

\section{TABLES}

Table 1. Plant species collected in the floating-leafed and submersed-macrophyte zones during survey of Shingobee and Williams Lakes

2. Sampled biomass by species for each depth on Shingobee Lake transects ...........13

3. Sampled biomass by species for each depth on Williams Lake transects............. 17

4. Total biomass collected during survey for each species found in Shingobee and Williams Lakes.

5. Biomass collected during survey for each species at each sampled depth in Shingobee Lake 
6. Biomass collected during survey for each species at each sampled depth in Williams Lake

7. Total biomass of aquatic macrophytes for Williams and Shingobee Lakes calculated from sample data

8. Percentage of carbon (C) in selected species collected during survey of Shingobee and Williams Lakes.

9. Percentage of nitrogen $(N)$ in selected species collected during survey of Shingobee and Williams Lakes.

10. Percentage of hydrogen $(\mathrm{H})$ in selected species collected during survey of Shingobee and Williams Lakes. 30

11. Percentage of phosphorus $(\mathrm{P})$ in selected species collected during survey of Shingobee and Williams Lakes.

12. Percentage of potassium $(\mathrm{K})$ in selected species collected during survey of Shingobee and Williams Lakes.

13. Percentage of calcium (Ca) in selected species collected during survey of Shingobee and Williams Lakes

14. Percentage of magnesium $(\mathrm{Mg})$ in selected species collected during survey of Shingobee and Williams Lakes

15. Plant species identified in the emergent zone of Shingobee Lake 36

16. Plant species identified in the emergent zone of Williams Lake 37

17. Williams Lake hypsographic and depth-volume data 40

18. Shingobee Lake hypsographic and depth-volume data 40

19. Attenuation coefficients $\left(\mathrm{K}_{\mathrm{PAR}}\right)$ calculated from radiometer data collected in Williams and Shingobee Lakes in 1991 and Secchi depth readings made in 1990 


\section{CONVERSION FACTORS}

Multiply

meter $(\mathrm{m})$

square meter $\left(\mathrm{m}^{2}\right)$

gram (g) by

3.281

10.764

.03527

\section{To obtain}

foot

square foot

ounces

Temperature in degrees Celsius $\left({ }^{\circ} \mathrm{C}\right)$ can be converted to degrees Fahrenheit $\left({ }^{\circ} \mathrm{F}\right)$ as follows:

$$
{ }^{\circ} \mathrm{F}=9 / 5\left({ }^{\circ} \mathrm{C}\right)+32
$$

Sea level: In this report "sea level" refers to the National Geodetic Vertical Datum of 1929 (NGVD of 1929)-a geodetic datum derived from a general adjustment of the first-order level nets of both the United States and Canada, formerly called Sea Level Datum of 1929. 


\title{
AQUATIC MACROPHYTES AND SELECTED PHYSICAL PROPERTIES OF SHINGOBEE AND WILLIAMS LAKES, MINNESOTA, 1991-92
}

by Virginia Carter, P. T. Gammon, D. O. Rosenberry, and Michael Turtora.

\begin{abstract}
This report summarizes data collected on the species composition and biomass of aquatic macrophytes in the floating-leafed and submersed aquatic zones of Shingobee and Williams Lakes in Minnesota. Plant species were identified and sampled on transects in each lake in August of 1991, and dry weights were determined for all samples. Nutrient contents were analysed for selected species. Data also were collected on the composition of species in the emergent zone of both lakes in June and August 1992. Light penetration was measured with an underwater spectroradiometer in August 1991. Hypsographic data were obtained and depth-volume calculations were made for both lakes.
\end{abstract}

\section{INTRODUCTION}

Williams and Shingobee Lakes, small lakes in north-central Minnesota, are two of several natural lakes selected by the U. S. Geological Survey and its cooperators for intensive field research on the interactions of the hydrologic cycle and nutrient balances of lakes (Siegal and Winter, 1980; LaBaugh and others, 1981). One of the objectives of the ongoing investigations at these lakes is to interrelate the physical and chemical characteristics of the water and biological production. To evaluate the species composition and biomass of aquatic macrophytes in these lakes, surveys were conducted in August 1991, and June and August 1992. This report presents the findings of these surveys and related information on Shingobee and Williams Lakes.

\section{ACKNOWLEDGMENTS}

We appreciate the assistance of Robert G. Striegl, Jr., U.S. Geological Survey, whose diving skills were required to sample the aquatic macrophytes, and Sharon Locke, University of Minnesota, who participated in the sample collection and handling.

\section{DESCRIPTION OF STUDY AREA}

Williams and Shingobee Lakes are in Hubbard County, Minnesota, about 150 miles northnortheast of Minneapolis-St. Paul and about 40 miles south-southeast of Bemidji (fig. 1). The surficial geologic materials in the Williams Lake drainage basin are mostly sand and gravel. Although the Minnesota Soil Atlas (Arneman and others, 1969) shows Williams Lake on a small northern projection of the Park Rapids-Staples glacial outwash plain, the local physiography is characteristic of 


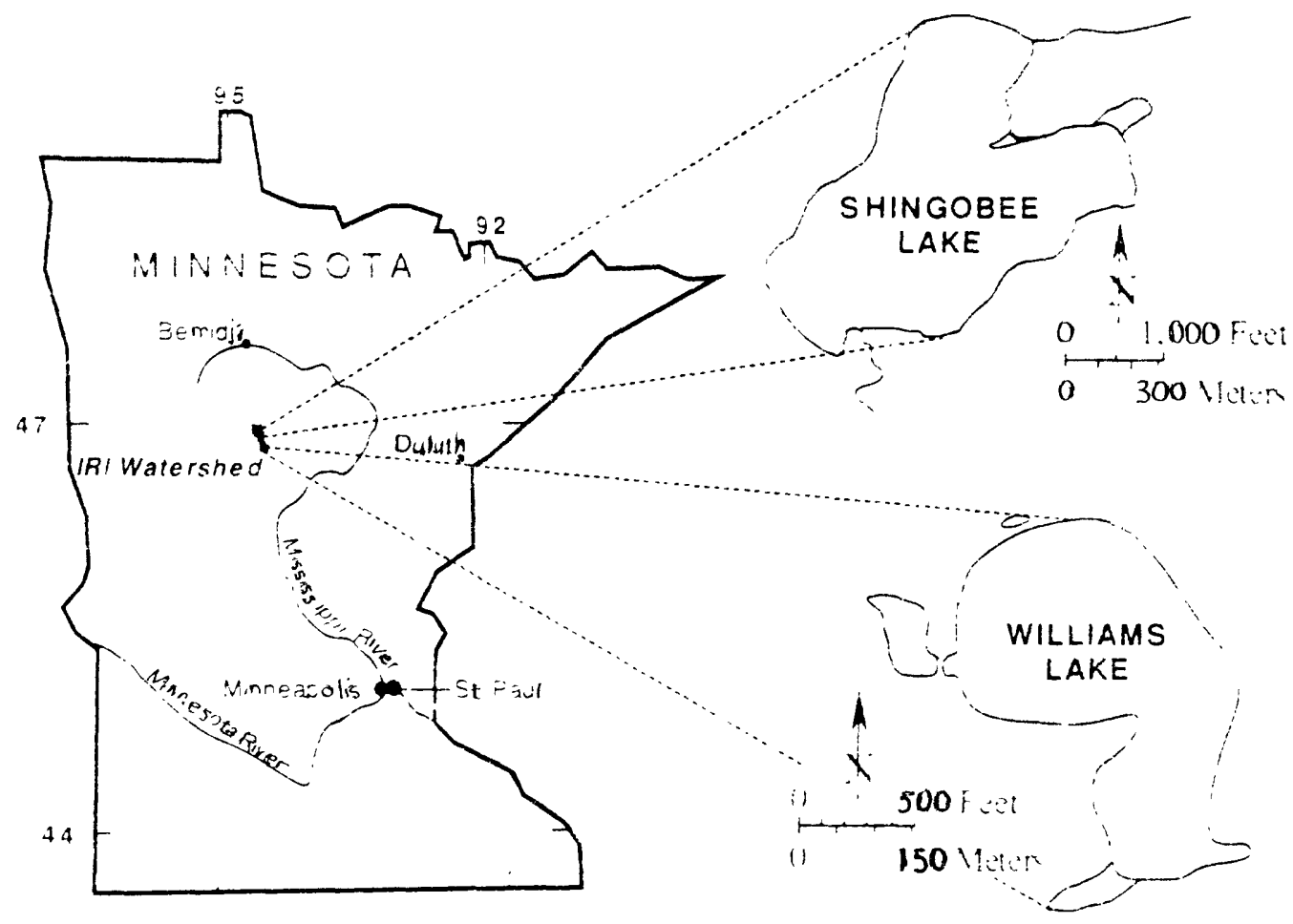

Figure 1. Map of the study area and lakes. 
ice-contact deposits (Siegel and Winter, 1980). Shingobee Lake is located at the southern edge of the Itasca Moraine; it lies north of, and at a lower elevation than Williams Lake.

No streams flow in or out of Williams Lake. Annual precipitation approximately balances evaporation (Kohler and others, 1959; Siegel and Winter, 1980), and ground water fluxes largely determine the annual hydrological balance. Shingobee Lake, on the other hand, has both an inlet and an outlet stream. Water-residence time in Williams Lake is about 3.7 years whereas water-residence time is about seven months in Shingobee Lake. Ground water in the region is approximately saturated with respect to calcite because of dissolution of soil carbonates by soil carbon dioxide (Groschen, 1981). Calcium and bicarbonate are the dominant ions in the lakes and in ground water. A survey of the aquatic macrophyte distribution in Williams Lake conducted in 1982 showed that 55 percent of the total lake area was covered by macrophytes (Taylor and others, 1985). Surveys of Shingobee Lake conducted by the Minnesota Department of Natural Resources in 1976 and 1989 noted species composition and abundance for submersed and emergent macrophytes (Minnesota Department of Natural Resources, written commun., 1992).

\section{COLLECTION AND ANALYSIS OF AQUATIC MACROPHYTE AND PHYSICAL PROPERTY DATA}

A survey was conducted to analyze species composition and determine biomass of submersed aquatic vegetation in Williams and Shingobee Lakes in August, 1991. Six transects were sampled in each lake (figs 2 and 3 ). Transects were perpendicular to the shore across the floating-leafed zone to the depth at which no appreciable amount of vegetation was present. Samples were collected at depths of $0.5,1,2,3,4$, and 5 meters in Shingobee Lake and at depths of $0.5,1,2,3,4,5,6$, and 7 meters in Williams Lake. At each depth, three 0.305 -m square (1-ft square) quadrats were randomly placed on the bottom and all vegetation within the quadrat was collected by divers. In the deeper sections where vegetation became sparse, quadrats were not placed randomly but an attempt was made to sample available plant material.

Each sample was sorted by species and each species placed in individual plastic mesh bags. Species were identified according to Fassett (1969) and Fernald (1970). (Appendix 1, table 1). Because of the heavy incrustation of calcium carbonate $\left(\mathrm{CaCO}_{3}\right)$ on the submersed plants, the samples were soaked in $100 \mathrm{mM}$ (millimolar) acetic acid for 12 to 16 hours to dissolve the carbonate. The floating-leafed and emergent species which had only small amounts of $\mathrm{CaCO}_{3}$ on the undersurfaces of the leaves, were not soaked. Samples were air dried for approximately 12 hours, after which they were dried in a $110^{\circ} \mathrm{C}$ (degrees Celsius) oven for 12 to 24 hours to determine dry weight in grams (g). Appendix 1, tables 2 and 3 , list sampled biomass by species for each depth in Shingobee and Williams Lakes. Appendix 1, table 4, lists total biomass for each species by lake. Appendix 1, tables 5 and 6 , list total biomass by species for each sampled depth in Shingobee and Williams Lakes. The hypsographic data in Appendix 4, tables 17 and 18, were used to calculate the area between metric contours. Appendix 1, table 9, lists estimates of total biomass for each lake based on these areas and sample data. 


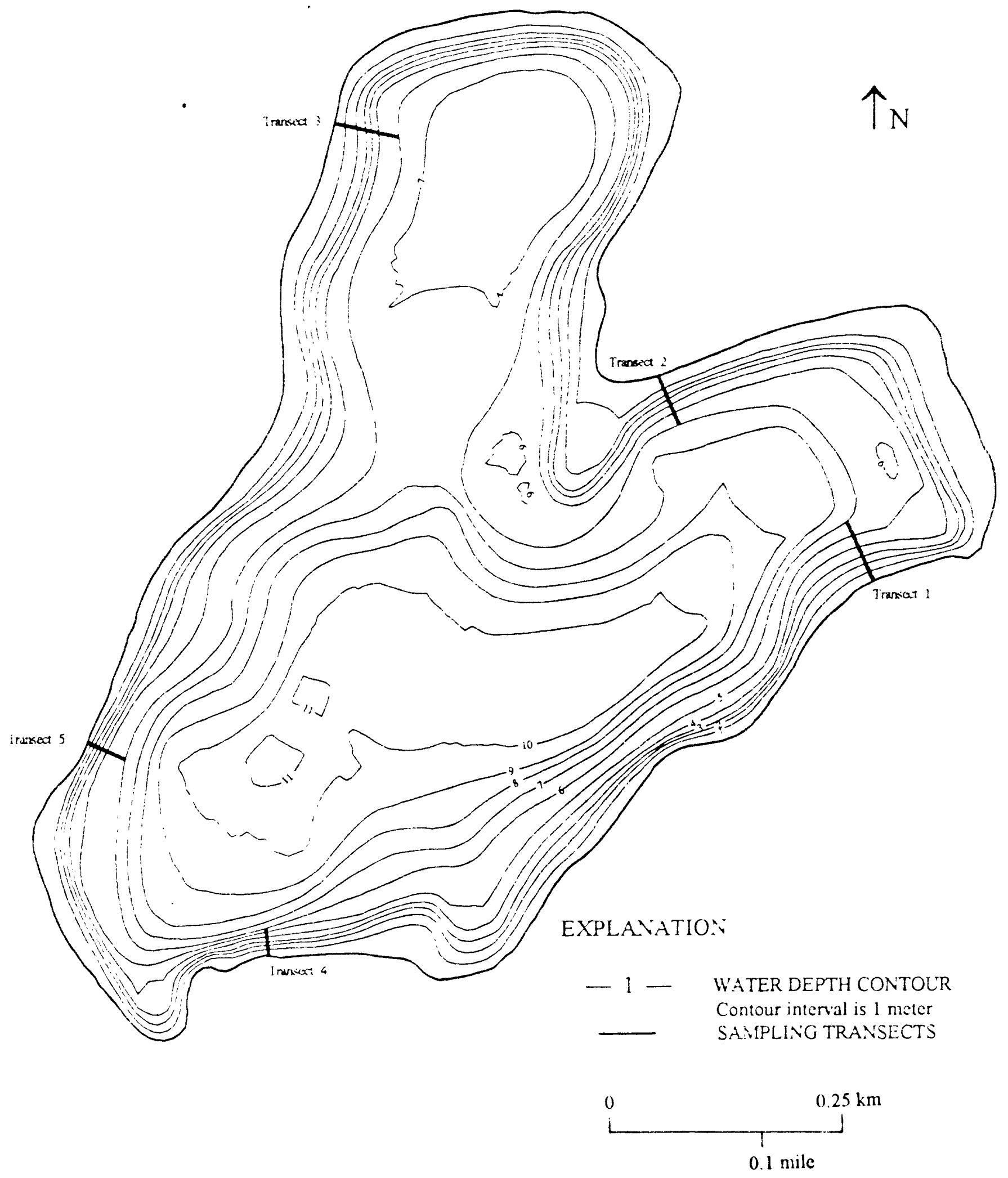

Figure 2 Bathymetric map of Shingobee Lake showing location of transects 


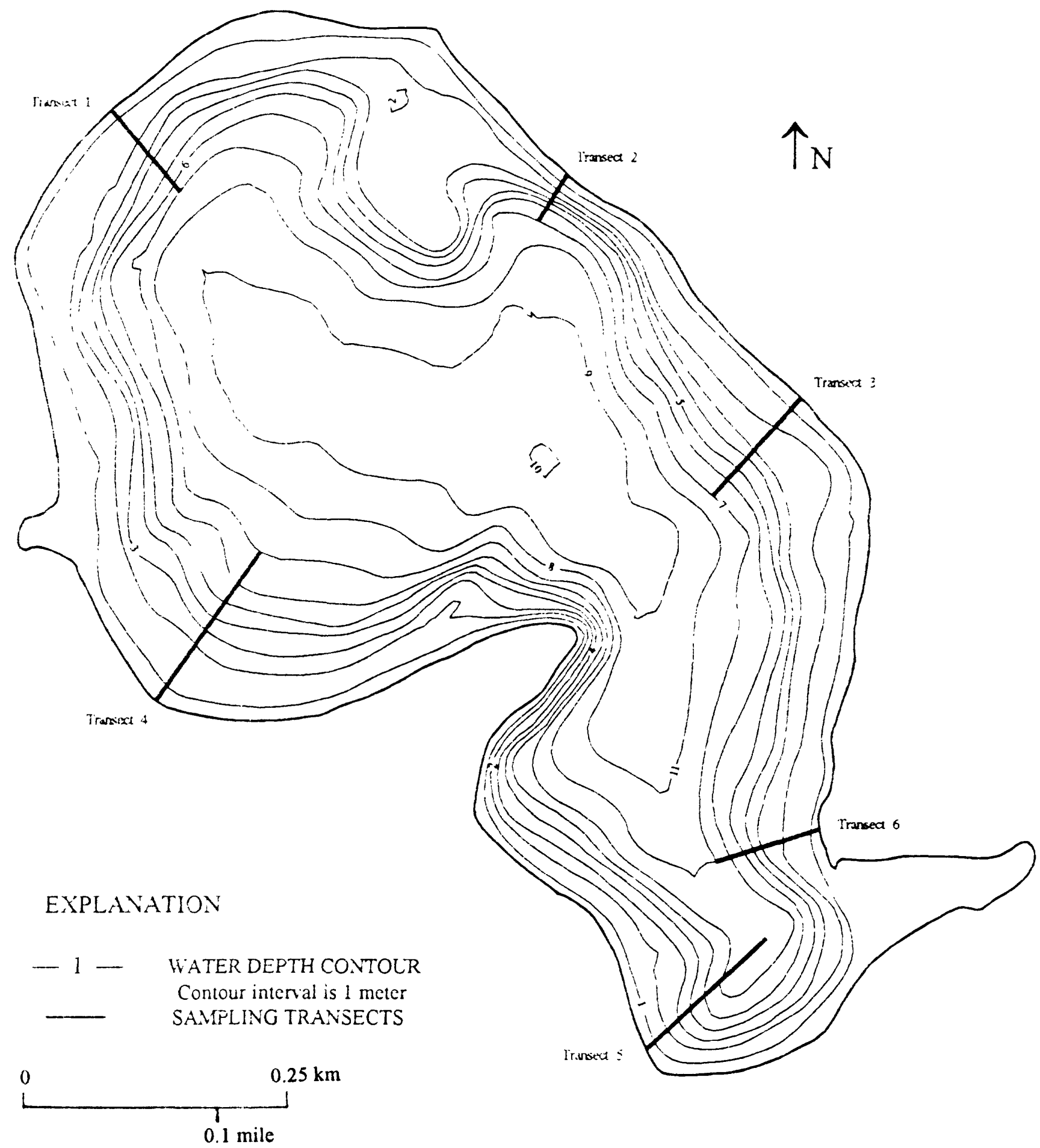

Figure 3. Bathymetric map of Williams Lake showing location of transects. 
Selected samples of plant material were sent to the University of Maryland Cooperative Extension Service for analysis of elemental nutrients. Percent by weight of carbon, hydrogen and nitrogen was determined using a Leco ${ }^{1} \mathrm{CHN}-600$ analyzer. Infrared absorption was used to determine carbon and hydrogen, and thermal conductivity was used to determine nitrogen. Percent by weight of phosphorus, potassium, calcium and magnesium was determined by digesting a 0.33 gram sample with nitric and perchloric acids; analysis was done on a Technicon AutoAnalyzer. Phosphorus and magnesium were determined using a colorimeter and potassium and calcium were determined using a flame photometer (Buriel, 1991, written commun.) (Appendix 2, tables 8 through 14).

In June and August 1992, a survey was made of the species composition of the emergent zone of both lakes (Appendix 3, tables 15 and 16). Species identification follows Fernald (1970), Voss (1972), and Britton and Brown (1970).

Hypsographic data for Williams Lake were obtained from a 0.616-m (2-ft)-interval bathymetric map of the lake (Minnesota Department of Natural Resources). Areas encircled by each 0.616-m (2-ft) contour were determined by planimetry, and depth-area data were interpolated to yield areas for each $1-\mathrm{m}$ stratum of the lake. The area of the lake was assumed to be zero at the 9.8 -meter depth; lake surface elevation is $421.38 \mathrm{~m}$ above sea level. For Shingobee Lake, bathymetric data were available on a 1.524-m (5-ft) interval (Minnesota Department of Natural Resources), and identical procedures were used to obtain hypsographic data for each $1-\mathrm{m}$ stratum. The area of the lake was assumed to be zero at $11.9-\mathrm{m}$ depth, and the elevation at the lake surface is $405.10 \mathrm{~m}$ above sea level.

Volumes for each stratum were calculated using the equation for a truncated cone:

$$
V=1 / 3\left(A_{T}+A_{B}+\left(A_{T} A_{B}\right)^{0.5}\right)(B-T)
$$

where

$\mathrm{A}_{\mathrm{T}} \quad=$ area at the top of each stratum,

$\mathrm{A}_{\mathrm{B}}=$ area at the bottom of each stratum, and

$\mathrm{B}-\mathrm{T}=$ difference between the depths at the bottom and top of each stratum.

Hypsographic and depth-volume data are presented in Appendix 4, tables 17 and 18, and depthvolume curves are shown in figure 4.

Incident and underwater irradiance were measured with a portable submersible scanning spectroradiometer. The radiometer is equipped with a hemispherical silicon detector, a holographic grating monochrometer and filter wheel to select narrow bandwidths, and an internal computer that handles all data collection and storage. Light energy, in watts per square meter $\left(\mathrm{W} \mathrm{m}^{-2}\right)$, was

\footnotetext{
${ }^{1}$ Brand names used in this report are for identification only and do not constitute endorsement by the U.S. Geological Survey.
} 


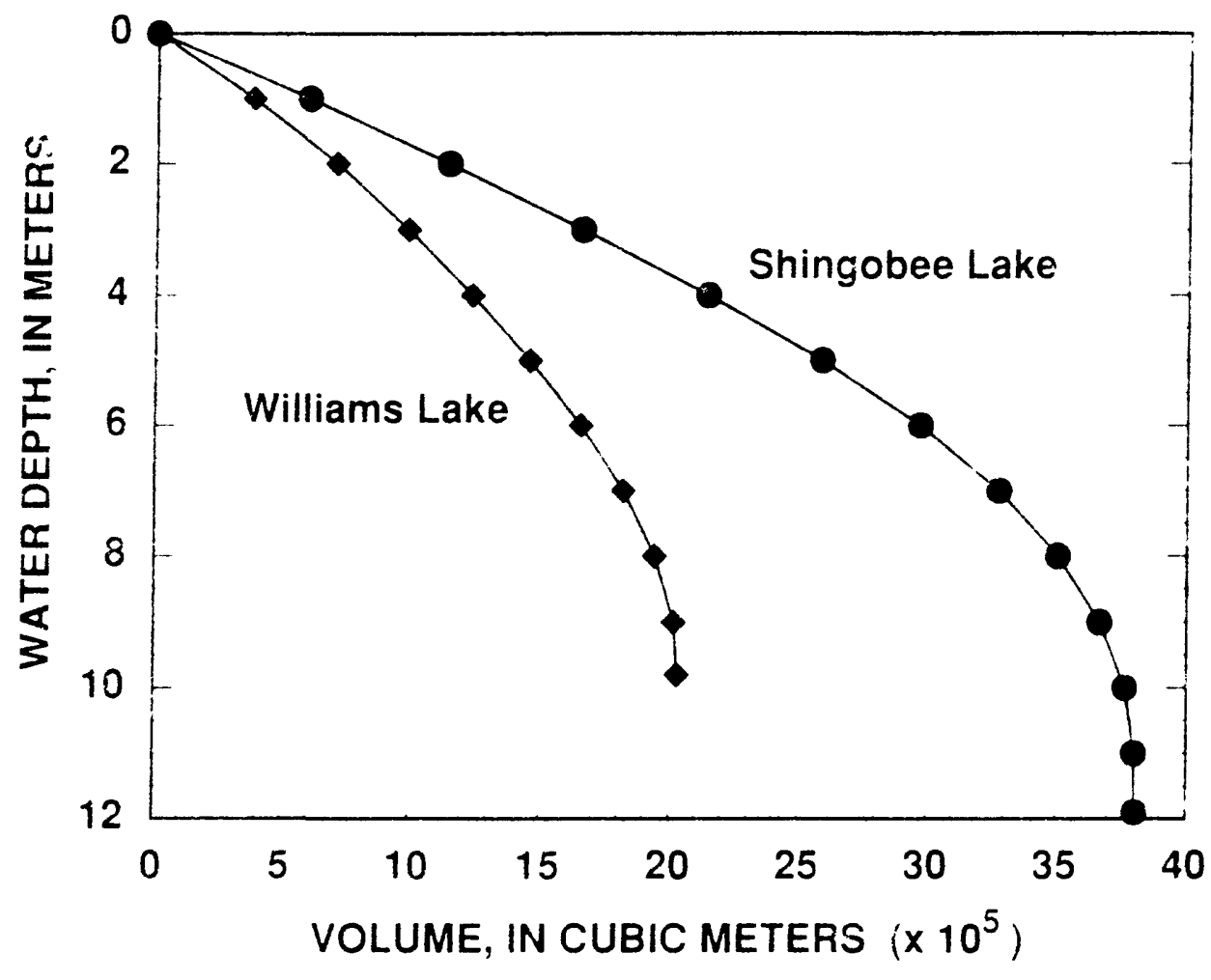

Figure 4. Depth-volume curves for Williams and Shingobee Lakes. 
measured at 5-nm (nanometer) light intervals from 400 to $800 \mathrm{~nm}$; each measurement represents the average of 5 complete scans. Measurements were made about $1 \mathrm{~m}$ from the boat in the center of the lake between 10:00 am and 2:00 pm Attenuation coefficients $\left(\mathrm{K}_{\lambda}\right)$ were calculated for all individual wavelengths measured and for total photosynthetically active radiation $\left(\mathrm{K}_{\mathrm{PAR}}, 400-700 \mathrm{~nm}\right)$ using Beer's Law:

$$
I_{z}=I_{0} e^{-K z},
$$

where

$\mathrm{I}_{\mathrm{z}}=$ average irradiance at depth $\mathrm{z}$, in $\mathrm{W} \mathrm{m}^{-2}$,

$I_{\mathrm{o}}=$ average light intensity just below the water surface, and

$\mathrm{K}=$ vertical attenuation coefficient $\left(\mathrm{m}^{-1}\right)$.

Appendix 5, table 19 lists attenuation coefficients $\left(\mathrm{K}_{\mathrm{PAR}}\right)$ for both lakes for selected depths. 


\section{REFERENCES}

Arneman, H.F., and others, 1969, Minnesota Soil Atlas, Brainerd Sheet: University of Minnesota Agricultural Experiment Station Miscellaneous Report 90-1969, 36 p.

Britton, N.L. and Brown, Addison, 1970, An illustrated flora of the northern United States and Canada: v. 1, New York, Dover Publications, Inc., 680 p.

Fassett, N.C., 1969, A manual of aquatic plants: University of Wisconsin Press, 405 p.

Fernald, M.L., 1970, Gray's manual of botany, eighth edition: D. Van Nostrand Company, 1632 p.

Groshen, G.E., 1981, Geochemistry of Williams Lake, Hubbard County, Minnesota: M.S. thesis, University of Minnesota, Minneapolis, $136 \mathrm{p}$.

Kohler, M.A., Nordenson, T.J., and Baker, D.R., 1959, Evaporation maps for the United States: U.S. Weather Bureau Technical Paper no. 37, 13 p.

LaBaugh, J.W., Groschen, G.E., and Winter, T.C., 1981, Limnological and geochemical survey of Williams Lake, Hubbard County, Minnesota: U.S. Geological Survey Water-Resources Investigations Report 81-41, 38 p.

Siegel, D.I., and Winter, T.C., 1980, Hydrologic setting of Williams Lake, Hubbard County, Minnesota: U.S. Geological Survey Open-File Report 80-403, 56 p.

Taylor, W. W., LaBaugh, J.W., Freeberg, M.H., and Dowling, D.C., 1985, Fishery survey and related limnological conditions of Williams Lake, Hubbard County, Minnesota: U.S. Geological Survey Water-Resources Investigations Report 84-4145, 25 p.

Voss, E.G., 1972, Michigan flora: part 1, gymnosperms and monocots: Cranbrook Institute of Science and the University of Michigan Herbarium, $488 \mathrm{p}$. 
Appendix 1. Aquatic Macrophyte Species and Biomass Data 
Table 1. Plant species collected in floating-leafed and submersed-macrophyte zones during survey of Shingobee and Williams Lakes

[Taxonomy follows Fernald (1970) and Fassett (1969)]

Lake

Species

Common name

\begin{tabular}{|c|c|c|}
\hline Shingobee & $\begin{array}{l}\text { Ceratophyllum demersum } \mathrm{L} . \\
\text { Chara sp. } \\
\text { Elodea canadensis Michx. } \\
\text { Lemna trisulca } \mathrm{L} \text {. } \\
\text { Myriophyllum exalbescens Fern. } \\
\text { Najas flexilis (Willd.) Rostk. \& Schmidt } \\
\text { Nuphar variegatum Engelm. } \\
\text { Nymphaea odorata Ait. } \\
\text { Potamogeton amplifolius } \text { Tuckerm. } \\
\text { Potamogeton natans } \mathrm{L} . \\
\text { Potamogeton pectinatus } \mathrm{L} . \\
\text { Potamogeton pulcher Tuckerm. } \\
\text { Potamogeton Richardsonii (Ar. Benn.) Rydb. } \\
\text { Potamogeton zosteriformis Fern. } \\
\text { Scirpus validus Vahl } \\
\text { Sparganium eurycarpum } \\
\text { Utricularia vulgaris } \mathrm{L} . \\
\text { Zizania aquatica } \mathrm{L} .\end{array}$ & $\begin{array}{l}\text { hornwort } \\
\text { muskgrass } \\
\text { waterweed } \\
\text { star duckweed } \\
\text { water-milfoil } \\
\text { naiad } \\
\text { bullhead lily } \\
\text { fragrant water-lily } \\
\text { large-leaf pondweed } \\
\text { pondweed } \\
\text { sago pondweed } \\
\text { pondweed } \\
\text { red-head pondweed } \\
\text { flat-stem pondweed } \\
\text { soft-stem bulrush } \\
\text { bur-reed } \\
\text { bladderwort } \\
\text { wild rice }\end{array}$ \\
\hline Williams & $\begin{array}{l}\text { Ceratophyllum demersum } \mathrm{L} . \\
\text { Chara sp. } \\
\text { Heteranthera dubia (Jacq.) MacM. } \\
\text { Megalodonta beckii (Torr.) Greene } \\
\text { Myriophyllum exalbescens Fern. } \\
\text { Najas flexilis (Willd.) Rostk. \& Schmidt } \\
\text { Nitella sp. } \\
\text { Nuphar variegatum Engelm. } \\
\text { Nymphaea odorata Ait. } \\
\text { Potamogeton amplifolius Tuckerm. } \\
\text { Potamogeton gramineus v. graminifolius Fries. } \\
\text { Potamogeton lucens L. } \\
\text { Potamogeton natans L. } \\
\text { Potamogeton pulcher Tuckerm. } \\
\text { Potamogeton Richardsonii (Ar. Benn) Rydb. } \\
\text { Potamogeton sp. L. } \\
\text { Potamogeton zosteriformis Fern. } \\
\text { Sagittaria graminea Michx. } \\
\text { Scirpus validus Vahl }\end{array}$ & $\begin{array}{l}\text { hornwort } \\
\text { muskgrass } \\
\text { water-stargrass } \\
\text { water marigold } \\
\text { water-milfoil } \\
\text { naiad } \\
\text { muskgrass } \\
\text { bullhead lily } \\
\text { fragrant water-lily } \\
\text { large-leaf pondweed } \\
\text { variable pondweed } \\
\text { pondweed } \\
\text { pondweed } \\
\text { pondweed } \\
\text { red-head pondweed } \\
\text { pondweed } \\
\text { flat-stem pondweed } \\
\text { arrowhead } \\
\text { soft-stem bulrush }\end{array}$ \\
\hline
\end{tabular}


Table 2. Sampled biomass by species for each depth on Shingobee Lake transects [depth in meters; biomass in grams; $\mathrm{t}=$ less than 0.05 gram]

\begin{tabular}{|c|c|c|c|}
\hline $\begin{array}{l}\text { Transect } \\
\text { number }\end{array}$ & Depth & Species & $\begin{array}{l}\text { Sampled } \\
\text { biomass }\end{array}$ \\
\hline 1 & 0.5 & Nymphaea odorata, Nuphar variegatum & 2.0 \\
\hline 1 & 0.5 & Sparganium eurycarpum & 13.3 \\
\hline 1 & 0.5 & Zizania aquatica & 5.1 \\
\hline 1 & 0.5 & Scirpus validus & $\mathrm{t}$ \\
\hline 1 & 0.5 & Ceratophyllum demersum & 25.3 \\
\hline 1 & 0.5 & Utricularia vulgaris & 0.6 \\
\hline 1 & 0.5 & Elodea canadensis & $\mathrm{t}$ \\
\hline 1 & 0.5 & Myriophyllum exalbescens & 0.2 \\
\hline 1 & 0.5 & Lemna trisulca & $\mathrm{t}$ \\
\hline 1 & 1 & Nymphaea odorata, Nuphar variegatum & 36.1 \\
\hline 1 & 1 & Sparganium eurycarpum & 1.3 \\
\hline 1 & 1 & Ceratophyllum demersum & 18.7 \\
\hline 1 & 1 & Potamogeton zosteriformis & 1.8 \\
\hline 1 & 1 & Utricularia vulgaris & 0.4 \\
\hline 1 & 1 & Potamogeton Richardsonii & $\mathrm{t}$ \\
\hline 1 & 2 & Ceratophyllum demersum & 20.3 \\
\hline 1 & 2 & Potamogeton zosteriformis & 3.8 \\
\hline 1 & 2 & Utricularia vulgaris & 0.2 \\
\hline 1 & 2 & Elodea canadensis & 0.1 \\
\hline 1 & 4 & Ceratophyllum demersum & 21.4 \\
\hline 1 & 4 & Potamogeton zosteriformis & 4.6 \\
\hline 1 & 4 & Potamogeton amplifolius & 1.4 \\
\hline 1 & 5 & Ceratophyllum demersum & 0.1 \\
\hline 2 & 0.5 & Nymphaea odorata, Nuphar variegatum & 0.3 \\
\hline 2 & 0.5 & Sparganium eurycarpum & 2.7 \\
\hline 2 & 0.5 & Scirpus validus & 20.1 \\
\hline 2 & 0.5 & Ceratophyllum demersum & 3.0 \\
\hline 2 & 0.5 & Myriophyllum exalbescens & 0.3 \\
\hline 2 & 0.5 & Najas flexilis & 0.1 \\
\hline 2 & 0.5 & Lemna trisulca & $\mathrm{t}$ \\
\hline 2 & 1 & Nymphaea odorata, Nuphar variegatum & 39.6 \\
\hline 2 & 1 & Scirpus validus & 12.1 \\
\hline 2 & 1 & Ceratophyllum demersum & 14.2 \\
\hline 2 & 1 & Potamogeton zosteriformis & 0.3 \\
\hline 2 & 1 & Utricularia vulgaris & 0.9 \\
\hline 2 & 1 & Myriophyllum exalbescens & $\mathrm{t}$ \\
\hline 2 & 2 & Ceratophyllum demersum & 72.3 \\
\hline 2 & 2 & Potamogeton zosteriformis & 0.7 \\
\hline 2 & 2 & Utricularia vulgaris & 4.4 \\
\hline
\end{tabular}


Table 2. Sampled biomass by species for each depth on Shingobee Lake transects -continued [depth in meters; biomass in grams; $t=$ less than 0.05 gram]

\begin{tabular}{|c|c|c|c|}
\hline $\begin{array}{l}\text { Transect } \\
\text { number }\end{array}$ & Depth & Species & $\begin{array}{l}\text { Sampled } \\
\text { biomass }\end{array}$ \\
\hline 2 & 2 & Myriophyllum exalbescens & 0.1 \\
\hline $\begin{array}{l}2 \\
2\end{array}$ & $\begin{array}{l}4 \\
4\end{array}$ & $\begin{array}{l}\text { Ceratophyllum demersum } \\
\text { Potamogeton zosteriformis }\end{array}$ & $\begin{array}{r}26.5 \\
2.6\end{array}$ \\
\hline $\begin{array}{l}2 \\
2 \\
2\end{array}$ & $\begin{array}{l}5 \\
5 \\
5\end{array}$ & $\begin{array}{l}\text { Nymphaea odorata, Nuphar variegatum } \\
\text { Ceratophyllum demersum } \\
\text { Potamogeton zosteriformis }\end{array}$ & $\begin{array}{r}\mathrm{t} \\
10.1 \\
0.7\end{array}$ \\
\hline $\begin{array}{l}3 \\
3 \\
3 \\
3 \\
3 \\
3 \\
3 \\
3 \\
3\end{array}$ & $\begin{array}{l}0.5 \\
0.5 \\
0.5 \\
0.5 \\
0.5 \\
0.5 \\
0.5 \\
0.5 \\
0.5\end{array}$ & $\begin{array}{l}\text { Nymphaea odorata, Nuphar variegatum } \\
\text { Nymphaea odorata, Nuphar variegatum } \\
\text { Sparganium eurycarpum } \\
\text { Scirpus validus } \\
\text { Ceratophyllum demersum } \\
\text { Myriophyllum exalbescens } \\
\text { Chara sp. } \\
\text { Potamogeton Richardsonii } \\
\text { Lemna trisulca }\end{array}$ & $\begin{array}{r}7.3 \\
7.3 \\
8.9 \\
\mathrm{t} \\
\mathrm{t} \\
\mathrm{t} \\
42.6 \\
\mathrm{t} \\
\mathrm{t}\end{array}$ \\
\hline $\begin{array}{l}3 \\
3 \\
3 \\
3 \\
3 \\
3\end{array}$ & $\begin{array}{l}1 \\
1 \\
1 \\
1 \\
1 \\
1\end{array}$ & $\begin{array}{l}\text { Nymphaea odorata, Nuphar variegatum } \\
\text { Ceratophyllum demersum } \\
\text { Myriophyllum exalbescens } \\
\text { Potamogeton amplifolius } \\
\text { Chara sp. } \\
\text { Potamogeton pectinatus }\end{array}$ & $\begin{array}{r}31.3 \\
1.2 \\
\mathrm{t} \\
1.0 \\
0.6 \\
0.8\end{array}$ \\
\hline $\begin{array}{l}3 \\
3 \\
3\end{array}$ & $\begin{array}{l}2 \\
2 \\
2\end{array}$ & $\begin{array}{l}\text { Ceratophyllum demersum } \\
\text { Potamogeton zosteriformis } \\
\text { Utricularia vulgaris }\end{array}$ & $\begin{array}{r}14.6 \\
\mathrm{t} \\
2.1\end{array}$ \\
\hline $\begin{array}{l}3 \\
3 \\
3 \\
3\end{array}$ & $\begin{array}{l}3 \\
3 \\
3 \\
3\end{array}$ & $\begin{array}{l}\text { Ceratophyllum demersum } \\
\text { Potamogeton zosteriformis } \\
\text { Chara sp. } \\
\text { Potamogeton pectinatus }\end{array}$ & $\begin{array}{r}47.4 \\
t \\
t \\
t\end{array}$ \\
\hline $\begin{array}{l}3 \\
3 \\
3\end{array}$ & $\begin{array}{l}4 \\
4 \\
4\end{array}$ & $\begin{array}{l}\text { Nymphaea odorata, Nuphar variegatum } \\
\text { Ceratophyllum demersum } \\
\text { Potamogeton zosteriformis }\end{array}$ & $\begin{array}{r}\mathrm{t} \\
5.0 \\
0.6\end{array}$ \\
\hline 3 & 5 & Ceratophyllum demersum & 0.1 \\
\hline $\begin{array}{l}4 \\
4 \\
4 \\
4 \\
4\end{array}$ & $\begin{array}{l}0.5 \\
0.5 \\
0.5 \\
0.5 \\
0.5\end{array}$ & $\begin{array}{l}\text { Nymphaea odorata, Nuphar variegatum } \\
\text { Sparganium eurycarpum } \\
\text { Scirpus validus } \\
\text { Ceratophyllum demersum } \\
\text { Potamogeton zosteriformis }\end{array}$ & $\begin{array}{r}28.5 \\
14.1 \\
17.8 \\
9.0 \\
\mathrm{t}\end{array}$ \\
\hline
\end{tabular}


Table 2. Sampled biomass by species for each depth on Shingobee Lake transects -continued [depth in meters; biomass in grams; $\mathrm{t}=$ less than 0.05 gram]

\begin{tabular}{|c|c|c|c|}
\hline $\begin{array}{l}\text { Transect } \\
\text { number }\end{array}$ & Depth & Species & $\begin{array}{c}\text { Sampled } \\
\text { biomass }\end{array}$ \\
\hline 4 & 0.5 & Utricularia vulgaris & 0.3 \\
\hline 4 & 0.5 & Elodea canadensis & $\mathrm{t}$ \\
\hline 4 & 0.5 & Chara sp. & 10.3 \\
\hline 4 & 0.5 & Potamogeton natans & 1.7 \\
\hline 4 & 1 & Nymphaea odorata, Nuphar variegatum & 49.6 \\
\hline 4 & 1 & Ceratophyllum demersum & 5.9 \\
\hline 4 & 1 & Potamogeton zosteriformis & $\mathrm{t}$ \\
\hline 4 & 1 & Utricularia vulgaris & 0.6 \\
\hline 4 & 1 & Myriophyllum exalbescens & 0.1 \\
\hline 4 & 1 & Chara sp. & 0.4 \\
\hline 4 & 1 & Potamogeton pectinatus & $\mathbf{t}$ \\
\hline 4 & 2 & Ceratophyllum demersum & 14.5 \\
\hline 4 & 2 & Potamogeton zosteriformis & $\mathbf{t}$ \\
\hline 4 & 2 & Utricularia vulgaris & 2.3 \\
\hline 4 & 2 & Potamogeton amplifolius & 0.4 \\
\hline 4 & 2 & Chara sp. & 0.4 \\
\hline 4 & 3 & Potamogeton zosteriformis & $\mathbf{t}$ \\
\hline 4 & 3 & Utricularia vulgaris & 1.2 \\
\hline 4 & 4 & Ceratophyllum demersum & 19.0 \\
\hline 4 & 4 & Utricularia vulgaris & 3.3 \\
\hline 4 & 4 & Chara sp. & 0.6 \\
\hline 4 & 4 & Lemna trisulca & $\mathbf{t}$ \\
\hline 4 & 5 & Ceratophyllum demersum & 0.6 \\
\hline 5 & 0.5 & Nymphaea odorata, Nuphar variegatum & 17.0 \\
\hline 5 & 0.5 & Sparganium eurycarpum & 4.6 \\
\hline 5 & 0.5 & Scirpus validus & 10.9 \\
\hline 5 & 0.5 & Ceratophyllum demersum & 11.2 \\
\hline 5 & 0.5 & Elodea canadensis & $\mathrm{t}$ \\
\hline 5 & 0.5 & Myriophyllum exalbescens & 0.1 \\
\hline 5 & 0.5 & Potamogeton pectinatus & 0.1 \\
\hline 5 & 0.5 & Potamogeton natans & 0.3 \\
\hline 5 & 0.5 & Lemna trisulca & $\mathrm{t}$ \\
\hline 5 & 1 & Nymphaea odorata, Nuphar variegatum & 1.0 \\
\hline 5 & 1 & Scirpus validus & 14.9 \\
\hline 5 & 1 & Ceratophyllum demersum & 11.4 \\
\hline 5 & 1 & Potamogeton zosteriformis & $t$ \\
\hline 5 & 1 & Myriophyllum exalbescens & $\mathrm{t}$ \\
\hline 5 & 1 & Potamogeton natans & 0.4 \\
\hline
\end{tabular}


Table 2. Sampled biomass by species for each depth on Shingobee Lake transects -continued [depth in meters; biomass in grams; $t=$ less than 0.05 gram]

\begin{tabular}{|c|c|c|c|}
\hline $\begin{array}{l}\text { Transect } \\
\text { number }\end{array}$ & Depth & Species & $\begin{array}{l}\text { Sampled } \\
\text { biomass }\end{array}$ \\
\hline 5 & 2 & Nymphaea odorata, Nuphar variegatum & 1.2 \\
\hline 5 & 2 & Ceratophyllum demersum & 28.5 \\
\hline 5 & 2 & Potamogeton zosteriformis & $\mathrm{t}$ \\
\hline 5 & 2 & Potamogeton Richardsonii & 0.5 \\
\hline 5 & 3 & Ceratophyllum demersum & 22.5 \\
\hline 5 & 3 & Utricularia vulgaris & 0.7 \\
\hline 5 & 4 & Nymphaea odorata, Nuphar variegatum & 1.2 \\
\hline 5 & 4 & Ceratophyllum demersum & 18.4 \\
\hline 5 & 4 & Potamogeton zosteriformis & 1.9 \\
\hline 5 & 5 & Ceratophyllum demersum & 0.6 \\
\hline 6 & 0.5 & Nymphaea odorata, Nuphar variegatum & 2.0 \\
\hline 6 & 0.5 & Scirpus validus & 30.3 \\
\hline 6 & 0.5 & Ceratophyllum demersum & 2.5 \\
\hline 6 & 0.5 & Utricularia vulgaris & 0.1 \\
\hline 6 & 0.5 & Chara sp. & 62.3 \\
\hline 6 & 1 & Nymphaea odorata, Nuphar variegatum & 18.3 \\
\hline 6 & 1 & Scirpus validus & 0.6 \\
\hline 6 & 1 & Ceratophyllum demersum & 9.2 \\
\hline 6 & 1 & Potamogeton zosteriformis & $\mathbf{t}$ \\
\hline 6 & 1 & Utricularia vulgaris & 0.3 \\
\hline 6 & 1 & Myriophyllum exalbescens & 0.3 \\
\hline 6 & 1 & Potamogeton pulcher & 0.2 \\
\hline 6 & 2 & Nymphaea odorata, Nuphar variegatum & 2.5 \\
\hline 6 & 2 & Ceratophyllum demersum & 10.5 \\
\hline 6 & 2 & Potamogeton zosteriformis & $\mathrm{t}$ \\
\hline 6 & 2 & Utricularia vulgaris & 2.9 \\
\hline 6 & 2 & Potamogeton pulcher & 0.8 \\
\hline 6 & 3 & Ceratophyllum demersum & 15.3 \\
\hline 6 & 3 & Potamogeton zosteriformis & 0.6 \\
\hline 6 & 3 & Potamogeton Richardsonii & 0.8 \\
\hline 6 & 4 & Ceratophyllum demersum & 7.6 \\
\hline 6 & 4 & Utricularia vulgaris & $\mathrm{t}$ \\
\hline 6 & 5 & Ceratophyllum demersum & 1.4 \\
\hline
\end{tabular}


Table 3. Sampled biomass by species for each depth on Williams Lake transects [depth in meters; biomass in grams; $\mathrm{t}=$ less than 0.05 gram]

\begin{tabular}{|c|c|c|c|}
\hline $\begin{array}{l}\text { Transect } \\
\text { number }\end{array}$ & Depth & Species & $\begin{array}{l}\text { Sampled } \\
\text { biomass }\end{array}$ \\
\hline 1 & 0.5 & Nymphaea odorata, Nuphar variegatum & 9.9 \\
\hline 1 & 0.5 & Potamogeton zosteriformis & 4.2 \\
\hline 1 & 0.5 & Myriophyllum exalbescens & 1.3 \\
\hline 1 & 0.5 & Potamogeton pectinatus & 0.1 \\
\hline 1 & 0.5 & Potamogeton natans & 1.5 \\
\hline 1 & 0.5 & Najas flexilis & 2.7 \\
\hline 1 & 0.5 & Heteranthera dubia & 1.0 \\
\hline 1 & 0.5 & Megalodonta beckii & 0.2 \\
\hline 1 & 1 & Nymphaea odorata, Nuphar variegatum & 15.0 \\
\hline 1 & 1 & Ceratophyllum demersum & $\mathbf{t}$ \\
\hline 1 & 1 & Potamogeton zosteriformis & 0.3 \\
\hline 1 & 1 & Myriophyllum exalbescens & 8.8 \\
\hline 1 & 1 & Potamogeton amplifolius & 1.4 \\
\hline 1 & 1 & Potamogeton natans & 0.1 \\
\hline 1 & 1 & Najas flexilis & $\mathbf{t}$ \\
\hline 1 & 1 & Heteranthera dubia & 19.6 \\
\hline 1 & 1 & Megalodonta beckii & 0.2 \\
\hline 1 & 2 & Ceratophyllum demersum & 0.4 \\
\hline 1 & 2 & Potamogeton zosteriformis & 17.3 \\
\hline 1 & 2 & Myriophyllum exalbescens & 0.8 \\
\hline 1 & 2 & Potamogeton amplifolius & 1.5 \\
\hline 1 & 2 & Potamogeton pectinatus & 4.9 \\
\hline 1 & 2 & Heteranthera dubia & 26.7 \\
\hline 1 & 2 & Megalodonta beckii & 28.7 \\
\hline 1 & 3 & Ceratophyllum demersum & 0.3 \\
\hline 1 & 3 & Potamogeton zosteriformis & 8.4 \\
\hline 1 & 3 & Myriophyllum exalbescens & 4.2 \\
\hline 1 & 3 & Potamogeton amplifolius & 2.7 \\
\hline 1 & 3 & Megalodonta beckii & 12.3 \\
\hline 1 & 4 & Potamogeton zosteriformis & 0.4 \\
\hline 1 & 4 & Potamogeton amplifolius & 5.2 \\
\hline 1 & 4 & Chara sp. & 10.8 \\
\hline 1 & 4 & Heteranthera dubia & $\mathrm{t}$ \\
\hline 1 & 4 & Megalodonta beckii & 6.5 \\
\hline 1 & 5 & Potamogeton zosteriformis & $\mathbf{t}$ \\
\hline 1 & 5 & Potamogeton amplifolius & 4.0 \\
\hline 1 & 5 & Megalodonta beckii & 0.3 \\
\hline 1 & 6 & Ceratophyllum demersum & 50.6 \\
\hline
\end{tabular}


Table 3. Sampled biomass by species for each depth on Williams Lake transects -continued [depth in meters; biomass in grams; $\mathrm{t}=$ less than $0.05 \mathrm{~g}$ ]

\begin{tabular}{|c|c|c|c|}
\hline $\begin{array}{l}\text { Transect } \\
\text { number }\end{array}$ & Depth & Species & $\begin{array}{l}\text { Sampled } \\
\text { biomass }\end{array}$ \\
\hline 2 & 0.5 & Sagittaria graminea & 0.7 \\
\hline 2 & 0.5 & Ceratophyllum demersum & $\mathrm{t}$ \\
\hline 2 & 0.5 & Potamogeton amplifolius & 4.2 \\
\hline 2 & 0.5 & Potamogeton pectinatus & 0.2 \\
\hline 2 & 0.5 & Najas flexilis & 0.8 \\
\hline 2 & 0.5 & Heteranthera dubia & 1.0 \\
\hline 2 & 1 & Potamogeton zosteriformis & 6.6 \\
\hline 2 & 1 & Myriophyllum exalbescens & 0.2 \\
\hline 2 & 1 & Potamogeton amplifolius & 2.7 \\
\hline 2 & 1 & Potamogeton pectinatus & 0.8 \\
\hline 2 & 1 & Najas flexilis & $t$ \\
\hline 2 & 1 & Heteranthera dubia & 0.2 \\
\hline 2 & 1 & Megalodonta beckii & 1.0 \\
\hline 2 & 2 & Potamogeton zosteriformis & 2.4 \\
\hline 2 & 2 & Potamogeton pectinatus & 7.7 \\
\hline 2 & 2 & Megalodonta beckii & 22.5 \\
\hline 2 & 3 & Ceratophyllum demersum & 0.1 \\
\hline 2 & 3 & Myriophyllum exalbescens & 2.6 \\
\hline 2 & 3 & Potamogeton amplifolius & 2.8 \\
\hline 2 & 3 & Potamogeton pectinatus & 3.0 \\
\hline 2 & 3 & Heteranthera dubia & 3.0 \\
\hline 2 & 3 & Megalodonta beckii & 3.0 \\
\hline 2 & 4 & Ceratophyllum demersum & $\mathbf{t}$ \\
\hline 2 & 4 & Myriophyllum exalbescens & 1.8 \\
\hline 2 & 4 & Potamogeton amplifolius & 12.3 \\
\hline 2 & 4 & Heteranthera dubia & 6.2 \\
\hline 2 & 5 & Myriophyllum exalbescens & 0.8 \\
\hline 2 & 5 & Potamogeton amplifolius & 1.1 \\
\hline 2 & 6 & Potamogeton zosteriformis & 0.2 \\
\hline 2 & 6 & Myriophyllum exalbescens & 0.2 \\
\hline 2 & 6 & Potamogeton amplifolius & $\mathbf{t}$ \\
\hline 3 & 0.5 & Chara sp. & 8.9 \\
\hline 3 & 0.5 & Najas flexilis & 0.1 \\
\hline 3 & 0.5 & Potamogeton gramineus var graminifolius & $\mathbf{t}$ \\
\hline 3 & 1 & Sagittaria graminea & 2.0 \\
\hline 3 & 1 & Potamogeton zosteriformis & 0.3 \\
\hline 3 & 1 & Myriophyllum exalbescens & 0.8 \\
\hline 3 & 1 & Potamogeton amplifolius & 1.8 \\
\hline
\end{tabular}


Table 3. Sampled biomass by species for each depth on Williams Lake transects -continued [depth in meters; biomass in grams; $\mathrm{t}=$ less than $0.05 \mathrm{~g}$ ]

\begin{tabular}{|c|c|c|c|}
\hline $\begin{array}{l}\text { Transect } \\
\text { number }\end{array}$ & Depth & Species & $\begin{array}{c}\text { Sampled } \\
\text { biomass }\end{array}$ \\
\hline 3 & 1 & Chara sp. & 4.0 \\
\hline 3 & 1 & Najas flexilis & 0.6 \\
\hline 3 & 1 & Megalodonta beckii & 0.2 \\
\hline 3 & 1 & Potamogeton gramineus var graminifolius & 0.5 \\
\hline 3 & 1 & Potamogeton lucens & 0.8 \\
\hline 3 & 2 & Myriophyllum exalbescens & 0.5 \\
\hline 3 & 2 & Chara sp. & 47.5 \\
\hline 3 & 2 & Potamogeton lucens & 1.8 \\
\hline 3 & 3 & Potamogeton zosteriformis & 24.5 \\
\hline 3 & 3 & Myriophyllum exalbescens & 1.6 \\
\hline 3 & 3 & Potamogeton amplifolius & 13.2 \\
\hline 3 & 3 & Chara sp. & $\mathbf{t}$ \\
\hline 3 & 3 & Mixed $P$. zosteriformis and Chara sp. & 13.8 \\
\hline 3 & 4 & Potamogeton zosteriformis & $\mathrm{t}$ \\
\hline 3 & 4 & Myriophyllum exalbescens & 1.4 \\
\hline 3 & 4 & Potamogeton amplifolius & 4.2 \\
\hline 3 & 4 & Chara sp. & 40.0 \\
\hline 3 & 5 & Myriophyllum exalbescens & 0.4 \\
\hline 3 & 5 & Potamogeton amplifolius & $\mathbf{t}$ \\
\hline 3 & 5 & Potamogeton pectinatus & 0.1 \\
\hline 3 & 5 & Heteranthera dubia & 0.1 \\
\hline 3 & 6 & Ceratophyllum demersum & 0.1 \\
\hline 3 & 6 & Myriophyllum exalbescens & 0.2 \\
\hline 3 & 6 & Potamogeton sp. & $\mathbf{t}$ \\
\hline 4 & 0.5 & Sagittaria graminea & 0.1 \\
\hline 4 & 0.5 & Potamogeton zosteriformis & 1.0 \\
\hline 4 & 0.5 & Myriophyllum exalbescens & 1.9 \\
\hline 4 & 0.5 & Chara sp. & 6.2 \\
\hline 4 & 0.5 & Najas flexilis & 0.9 \\
\hline 4 & 0.5 & Megalodonta beckii & 0.2 \\
\hline 4 & 1 & Nymphaea odorata, Nuphar variegatum & 5.0 \\
\hline 4 & 1 & Sagittaria graminea & $\mathrm{t}$ \\
\hline 4 & 1 & Myriophyllum exalbescens & 3.9 \\
\hline 4 & 1 & Chara sp. & 4.9 \\
\hline 4 & 1 & Potamogeton pectinatus & 6.1 \\
\hline 4 & 1 & Potamogeton pulcher & 0.9 \\
\hline 4 & 1 & Potamogeton natans & 1.1 \\
\hline 4 & 1 & Najas flexilis & 7.4 \\
\hline
\end{tabular}


Table 3. Sampled biomass by species for each depth on Williams Lake transects -continued [depth in meters; biomass in grams; $\mathrm{t}=$ less than $0.05 \mathrm{~g}$ ]

\begin{tabular}{|c|c|c|c|}
\hline $\begin{array}{l}\text { Transect } \\
\text { number }\end{array}$ & Depth & Species & $\begin{array}{l}\text { Sampled } \\
\text { biomass }\end{array}$ \\
\hline $\begin{array}{l}4 \\
4\end{array}$ & 1 & $\begin{array}{l}\text { Potamogeton } \mathrm{sp} . \\
\text { Potamogeton lucens }\end{array}$ & $\begin{array}{l}8.7 \\
14\end{array}$ \\
\hline $\begin{array}{l}4 \\
4 \\
4 \\
4 \\
4 \\
4\end{array}$ & $\begin{array}{l}2 \\
2 \\
2 \\
2 \\
2 \\
2\end{array}$ & $\begin{array}{l}\text { Potamogeton zosteriformis } \\
\text { Myriophyllum exalbescens } \\
\text { Potamogeton amplifolius } \\
\text { Potamogeton natans } \\
\text { Heteranthera dubia } \\
\text { Megalodonta beckii }\end{array}$ & $\begin{array}{r}0.8 \\
1.3 \\
4.8 \\
18.9 \\
5.3 \\
13.5\end{array}$ \\
\hline $\begin{array}{l}4 \\
4 \\
4 \\
4\end{array}$ & $\begin{array}{l}3 \\
3 \\
3 \\
3\end{array}$ & $\begin{array}{l}\text { Potamogeton zosteriformis } \\
\text { Myriophyllum exalbescens } \\
\text { Potamogeton amplifolius } \\
\text { Megalodonta beckii }\end{array}$ & $\begin{array}{r}1.1 \\
11.8 \\
4.5 \\
33.6\end{array}$ \\
\hline $\begin{array}{l}4 \\
4 \\
4 \\
4 \\
4\end{array}$ & $\begin{array}{l}4 \\
4 \\
4 \\
4 \\
4\end{array}$ & $\begin{array}{l}\text { Ceratophyllum demersum } \\
\text { Potamogeton zosteriformis } \\
\text { Myriophyllum exalbescens } \\
\text { Potamogeton amplifolius } \\
\text { Chara sp. }\end{array}$ & $\begin{array}{l}2.0 \\
0.1 \\
0.3 \\
2.5 \\
0.3\end{array}$ \\
\hline $\begin{array}{l}4 \\
4 \\
4\end{array}$ & $\begin{array}{l}5 \\
5 \\
5\end{array}$ & $\begin{array}{l}\text { Myriophyllum exalbescens } \\
\text { Potamogeton amplifolius } \\
\text { Megalodonta beckii }\end{array}$ & $\begin{array}{l}0.4 \\
2.9 \\
0.1\end{array}$ \\
\hline $\begin{array}{l}4 \\
4 \\
4 \\
4\end{array}$ & $\begin{array}{l}6 \\
6 \\
6 \\
6\end{array}$ & $\begin{array}{l}\text { Potamogeton zosteriformis } \\
\text { Myriophyllum exalbescens } \\
\text { Potamogeton amplifolius } \\
\text { Potamogeton gramineus var graminifolius }\end{array}$ & $\begin{array}{l}0.4 \\
0.2 \\
0.2 \\
0.2\end{array}$ \\
\hline $\begin{array}{l}5 \\
5 \\
5 \\
5 \\
5 \\
5 \\
5 \\
5\end{array}$ & $\begin{array}{l}0.5 \\
0.5 \\
0.5 \\
0.5 \\
0.5 \\
0.5 \\
0.5 \\
0.5\end{array}$ & $\begin{array}{l}\text { Nymphaea odorata, Nuphar variegatum } \\
\text { Sagittaria graminea } \\
\text { Myriophyllum exalbescens } \\
\text { Chara sp. } \\
\text { Potamogeton natans } \\
\text { Najas flexilis } \\
\text { Heteranthera dubia } \\
\text { Potamogeton gramineus var graminifolius }\end{array}$ & $\begin{array}{r}10.6 \\
2.2 \\
0.1 \\
\mathrm{t} \\
3.5 \\
4.2 \\
\mathrm{t} \\
0.7\end{array}$ \\
\hline $\begin{array}{l}5 \\
5 \\
5 \\
5 \\
5 \\
5\end{array}$ & $\begin{array}{l}1 \\
1 \\
1 \\
1 \\
1 \\
1\end{array}$ & $\begin{array}{l}\text { Nymphaea odorata, Nuphar variegatum } \\
\text { Myriophyllum exalbescens } \\
\text { Potamogeton amplifolius } \\
\text { Potamogeton natans } \\
\text { Najas flexilis } \\
\text { Heteranthera dubia }\end{array}$ & $\begin{array}{r}17.3 \\
3.0 \\
5.9 \\
15.6 \\
\mathrm{t} \\
1.1\end{array}$ \\
\hline
\end{tabular}


Table 3. Sampled biomass by species for each depth on Williams Lake transects -continued [depth in meters; biomass in grams; $\mathrm{t}=$ less than $0.05 \mathrm{~g}$ ]

\begin{tabular}{|c|c|c|c|}
\hline $\begin{array}{l}\text { Transect } \\
\text { number }\end{array}$ & Depth & Species & $\begin{array}{l}\text { Sampled } \\
\text { biomass }\end{array}$ \\
\hline 5 & 1 & Megalodonta beckii & 0.7 \\
\hline 5 & 3 & Potamogeton zosteriformis & 0.7 \\
\hline 5 & 3 & Myriophyllum exalbescens & 1.0 \\
\hline 5 & 3 & Potamogeton amplifolius & 6.2 \\
\hline 5 & 3 & Megalodonta beckii & 7.2 \\
\hline 5 & 4 & Myriophyllum exalbescens & 0.3 \\
\hline 5 & 5 & Ceratophyllum demersum & $\mathrm{t}$ \\
\hline 5 & 5 & Potamogeton zosteriformis & $\mathrm{t}$ \\
\hline 5 & 5 & Myriophyllum exalbescens & $\mathrm{t}$ \\
\hline 5 & 5 & Potamogeton sp. & $\mathrm{t}$ \\
\hline 5 & 6 & Ceratophyllum demersum & $\mathrm{t}$ \\
\hline 5 & 6 & Potamogeton zosteriformis & $\mathrm{t}$ \\
\hline 5 & 6 & Myriophyllum exalbescens & $\mathrm{t}$ \\
\hline 5 & 6 & Potamogeton sp. & $\mathrm{t}$ \\
\hline 6 & 0.5 & Nymphaea odorata, Nuphar variegatum & 11.9 \\
\hline 6 & 0.5 & Scirpus validus & 1.2 \\
\hline 6 & 0.5 & Sagittaria graminea & 1.8 \\
\hline 6 & 0.5 & Ceratophyllum demersum & $\mathbf{t}$ \\
\hline 6 & 0.5 & Potamogeton zosteriformis & 0.1 \\
\hline 6 & 0.5 & Myriophyllum exalbescens & 0.4 \\
\hline 6 & 0.5 & Potamogeton amplifolius & 0.4 \\
\hline 6 & 0.5 & Potamogeton natans & 7.9 \\
\hline 6 & 0.5 & Najas flexilis & 3.5 \\
\hline 6 & 0.5 & Potamogeton sp. & $\mathrm{t}$ \\
\hline 6 & 1 & Nymphaea odorata, Nuphar variegatum & 30.0 \\
\hline 6 & 1 & Potamogeton zosteriformis & $\mathrm{t}$ \\
\hline 6 & 1 & Myriophyllum exalbescens & 0.8 \\
\hline 6 & 1 & Potamogeton amplifolius & 3.9 \\
\hline 6 & 1 & Chara sp. & $t$ \\
\hline 6 & 1 & Potamogeton natans & 20.2 \\
\hline 6 & 1 & Najas flexilis & 0.4 \\
\hline 6 & 1 & Heteranthera dubia & 1.9 \\
\hline 6 & 1 & Megalodonta beckii & 0.3 \\
\hline 6 & 2 & Potamogeton zosteriformis & 5.1 \\
\hline 6 & 2 & Myriophyllum exalbescens & 3.1 \\
\hline 6 & 2 & Potamogeton amplifolius & 4.1 \\
\hline 6 & 2 & Potamogeton pectinatus & 0.6 \\
\hline 6 & 2 & Potamogeton natans & 4.0 \\
\hline
\end{tabular}


Table 3. Sampled biomass by species for each depth on Williams Lake transects -continued [depth in meters; biomass in grams; $\mathrm{t}=$ less than $0.05 \mathrm{~g}$ ]

\begin{tabular}{lrlr}
\hline $\begin{array}{c}\text { Transect } \\
\text { number }\end{array}$ & Depth & Species & $\begin{array}{r}\text { Sampled } \\
\text { biomass }\end{array}$ \\
\hline 6 & 2 & Najas flexilis & 0.1 \\
6 & 2 & Heteranthera dubia & 13.0 \\
6 & 2 & Megalodonta beckii & 4.9 \\
6 & 3 & Potamogeton zosteriformis & 3.3 \\
6 & 3 & Myriophyllum exalbescens & 1.0 \\
6 & 3 & Potamogeton amplifolius & 19.5 \\
6 & 3 & Heteranthera dubia & 10.7 \\
6 & 3 & Megalodonta beckii & 1.2 \\
& 4 & Potamogeton zosteriformis & 1.5 \\
6 & 4 & Myriophyllum exalbescens & 2.4 \\
6 & 4 & Potamogeton amplifolius & 5.6 \\
6 & 4 & Chara sp. & $\mathrm{t}$ \\
6 & 4 & Heteranthera dubia & 0.3 \\
6 & 4 & Megalodonta beckii & 1.8 \\
6 & 5 & Potamogeton zosteriformis & 0.4 \\
6 & 5 & Myriophyllum exalbescens & 1.5 \\
6 & 5 & Potamogeton sp. & 0.3 \\
6 & 5 & Nitella sp. & 2.5 \\
6 & 6 & Potamogeton zosteriformis & 0.1 \\
6 & 6 & Myriophyllum exalbescens & 0.7 \\
6 & 6 & Potamogeton sp. & 0.1 \\
6 & 7 & Nitella sp. & 0.5 \\
6 & & & \\
& & &
\end{tabular}


Table 4. Total biomass collected during survey for each species found in Shingobee and Williams Lakes

[leaders (-----) indicate species not found; biomass in grams]

\begin{tabular}{|c|c|c|}
\hline Species & $\begin{array}{r}\text { Shingobee } \\
\text { Lake } \\
\text { Biomass } \\
\end{array}$ & $\begin{array}{r}\text { Williams } \\
\text { Lake } \\
\text { Biomass } \\
\end{array}$ \\
\hline Ceratophyllum demersum & 491.5 & 53.5 \\
\hline Chara sp. & 117.2 & 122.7 \\
\hline Elodea canadensis & 0.2 & ----- \\
\hline Heteranthera dubia & ---- & 90.4 \\
\hline Lemna trisulca & 0.1 & $\ldots$ \\
\hline Megalodonta beckii & $-\cdots$ & 138.2 \\
\hline Mixed $P$. zosteriformis and Chara sp. & ----- & 13.8 \\
\hline Myriophyllum exalbescens & 1.1 & 59.5 \\
\hline Najas flexilis & 0.1 & 20.7 \\
\hline Nitella sp. & $-\cdots$ & 3.0 \\
\hline Nymphaea odorata, Nuphar variegatum & 238.1 & 99.7 \\
\hline Potamogeton Richardsonii & 1.4 & --- \\
\hline Potamogeton amplifolius & 2.7 & 117.3 \\
\hline Potamogeton gramineus v. graminifolius & ---- & 1.4 \\
\hline Potamogeton lucens & ---- & 4.1 \\
\hline Potamogeton natans & 2.4 & 72.8 \\
\hline Potamogeton pectinatus & 1.0 & 23.5 \\
\hline Potamogeton pulcher & 0.9 & 0.9 \\
\hline Potamogeton sp. & ---- & 9.2 \\
\hline Potamogeton zosteriformis & 18.0 & 79.3 \\
\hline Sagittaria graminea & ---- & 6.7 \\
\hline Scirpus validus & 106.8 & 1.2 \\
\hline Sparganium eurycarpum & 44.8 & $-\cdots$ \\
\hline Utricularia vulgaris & 20.2 & ---- \\
\hline Zizania aquatica & 5.1 & -..-- \\
\hline Total biomass & 1051.7 & 918.0 \\
\hline
\end{tabular}


Table 5. Biomass collected during survey for each species at each sampled depth in Shingobee Lake [biomass in grams; $\mathrm{t}=$ less than 0.05 ; leader (-.--) indicates species not found; Nym. $=$ Nymphaea; Nuph. $=$ Nuphar $]$

\begin{tabular}{|c|c|c|c|c|c|c|}
\hline \multirow[b]{2}{*}{ Species } & \multicolumn{6}{|c|}{ Depth in meters } \\
\hline & 0.5 & 1 & 2 & 3 & 4 & 5 \\
\hline Ceratophyllum demersum & 51.0 & 60.5 & 160.6 & 108.6 & 98.0 & 12.8 \\
\hline Chara sp. & 115.2 & 0.9 & 0.4 & $t$ & 0.6 & ---- \\
\hline Elodea canadensis & 0.1 &.--- & 0.1 & -...- & -..-- & ---- \\
\hline Lemna trisulca & 0.1 & $\cdots$ & $\cdots$ & -..-- & $\mathbf{t}$ & -..- \\
\hline Myriophyllum exalbescens & 0.6 & 0.4 & 0.1 & -...-- & --..- & $-\ldots$ \\
\hline Najas flexilis & 0.1 & $\cdots$ & ---- & ---- & $\cdots$ & --.- \\
\hline Nym. odorata, Nuph. variegatum & 57.2 & 176.0 & 3.7 & $\cdots-\cdots$ & 1.2 & $t$ \\
\hline Potamogeton Richardsonii & $t$ & $t$ & 0.5 & 0.8 & --- & $-\cdots$ \\
\hline Potamogeton amplifolius & ----- & 1.0 & 0.4 & $\cdots$ & 1.4 & --.- \\
\hline Potamogeton natans & 2.0 & 0.4 & $\cdots$ & $\cdots$ & $\cdots$ & ---- \\
\hline Potamogeton pectinatus & 0.1 & 0.9 & -.... & $t$ & $\ldots$ & -..-- \\
\hline Potamogeton pulcher & $\cdots$ & 0.2 & 0.8 & $\cdots$ & $\cdots$ & $-\cdots-$ \\
\hline Potamogeton zosteriformis & $t$ & 2.2 & 4.7 & 0.7 & 9.8 & 0.7 \\
\hline Scirpus validus & 79.2 & 27.7 & ---- & $\cdots$ & $\cdots$ & ---- \\
\hline Sparganium eurycarpum & 43.4 & 1.3 & -...- & -.-- & --.- & -...- \\
\hline Utricularia vulgaris & 0.9 & 2.1 & 11.9 & 2.0 & 3.3 & -...- \\
\hline Zizania aquatica & 5.1 & $\cdots$ & $\cdots$ & $-\cdots$ & $\cdots$ & $\cdots$ \\
\hline Total biomass & 355.0 & 273.6 & 183.1 & 112.1 & 114.3 & 13.5 \\
\hline
\end{tabular}


Table 6. Biomass collected during survey for each species at each sampled depth in Williams Lake [biomass in grams; $\mathrm{t}=$ less than 0.05 ; leader (---) indicates species not found; Nym. $=$ Nymphaea; Nup. = Nuphar]

\begin{tabular}{|c|c|c|c|c|c|c|c|c|}
\hline \multirow[b]{2}{*}{ Species } & \multicolumn{8}{|c|}{ Depth in meters } \\
\hline & 0.5 & 1 & 2 & 3 & 4 & 5 & 6 & 7 \\
\hline Megalodonta beckii & 0.4 & 2.3 & 69.6 & 57.3 & 8.3 & 0.3 & $\ldots$ & $\ldots$ \\
\hline Ceratophyllum demersum & $\mathbf{t}$ & $\mathbf{t}$ & 0.4 & 0.3 & 2.0 & $\mathbf{t}$ & 50.7 & $\ldots$ \\
\hline Chara sp. & 15.1 & 8.9 & 47.5 & $\mathbf{t}$ & 51.2 & $\ldots$ & -.. & $\ldots$ \\
\hline Heteranthera dubia & 2.1 & 22.9 & 44.9 & 13.7 & 6.6 & 0.1 & - & $\ldots$ \\
\hline Mixed $P$. zosteriformis/Chara sp. & - & $\cdots$ & -- & 13.8 & -- & -- & - & $-\ldots$ \\
\hline Myriophyllum exalbescens & 3.6 & 17.4 & 5.7 & 22.1 & 6.2 & 3.2 & 1.3 & - \\
\hline Najas flexilis & 12.1 & 8.5 & 0.1 & -- & $\cdots$ & $-\cdots$ & - & - \\
\hline Nitella sp. & -- & $\ldots$ & $-\ldots$ & - & -- & 2.5 & - & 0.5 \\
\hline Nym. odorata, Nup. variegatum & 32.4 & 67.3 & - & --- & --- & $\cdots$ & - & -- \\
\hline Potamogeton amplifolius & 4.6 & 15.7 & 10.4 & 48.8 & 29.7 & 8.0 & 0.2 & $\cdots$ \\
\hline P. gramineus v. graminifolius & 0.7 & 0.5 & $-\cdots$ & -- & -- & -- & 0.2 & - \\
\hline Potamogeton lucens & --- & 2.3 & 1.8 & $\cdots$ & $-\cdots$ & - & - & - \\
\hline Potamogeton natans & 12.9 & 37.0 & 22.9 & --- & -- & $-\cdots$ & -- & --- \\
\hline Potamogeton pectinatus & 0.3 & 6.8 & 13.2 & 3.0 & $\cdots$ & 0.1 & -- & -- \\
\hline Potamogeton pulcher & -- & 0.9 & --- & -- & - & - & $-\cdots$ & - \\
\hline Potamogeton sp. & -- & 8.7 & $\cdots$ & $-\cdots$ & - & 0.3 & 0.1 & $\ldots$ \\
\hline Potamogeton zosteriformis & 5.2 & 7.1 & 25.6 & 38.1 & 2.1 & 0.4 & 0.8 & - \\
\hline Sagittaria graminea & 4.7 & 2.0 & --- & -- & - & - & $-\cdots$ & $\cdots$ \\
\hline Scirpus validus & 1.2 & -- & - & - & $-\cdots$ & $\ldots$ & $\ldots$ & - \\
\hline Total biomass & 95.3 & 208.3 & 242.2 & 197.2 & 106.1 & 15.0 & 53.4 & 0.5 \\
\hline
\end{tabular}


Table 7. Total biomass of aquatic macrophytes for Williams and Shingobee Lakes calculated from sample data.

[ $\mathrm{m}^{2}=$ square meters; $\mathrm{g} \mathrm{ft}^{-1}=$ grams per square foot; $\mathrm{g} \mathrm{m}^{-2}=$ grams per square meter]

\begin{tabular}{lccccc}
\hline Lake & Depth & $\begin{array}{c}\text { Area } \\
\left(\mathrm{m}^{2} \times 10^{5}\right)\end{array}$ & $\begin{array}{c}\text { Mean biomass } \\
\left(\mathrm{g} \mathrm{ft}^{-1}\right)\end{array}$ & $\begin{array}{c}\text { Mean biomass } \\
\left(\mathrm{g} \mathrm{m}^{-2}\right)\end{array}$ & $\begin{array}{c}\text { Total biomass } \\
\left(\mathrm{g} \mathrm{x} \mathrm{10}^{5}\right)\end{array}$ \\
\hline Shingobee & & & & & \\
& $0-1$ & 0.575 & 59.17 & 636.91 & 366.22 \\
& $1-2$ & 0.419 & 38.06 & 409.68 & 171.66 \\
& $2-3$ & 0.246 & 29.53 & 317.86 & 78.19 \\
& $3-4$ & 0.302 & 22.65 & 243.80 & 73.63 \\
& $4-5$ & 0.492 & 11.62 & 125.08 & 61.54 \\
& $5-6$ & 0.742 & 2.70 & 29.06 & 21.56 \\
\hline & & & & Total & 772.80
\end{tabular}

Total biomass in metric tons $=77.28$

Williams

\begin{tabular}{lcccc}
$0-1$ & 0.375 & 15.89 & 171.04 & 64.14 \\
$1-2$ & 0.537 & 40.95 & 440.79 & 236.70 \\
$2-3$ & 0.432 & 39.94 & 429.91 & 185.72 \\
$3-4$ & 0.256 & 26.00 & 279.86 & 71.65 \\
$4-5$ & 0.260 & 10.38 & 111.73 & 29.05 \\
$5-6$ & 0.245 & 5.69 & 61.25 & 15.01 \\
$6-7$ & 0.395 & 7.70 & 82.88 & 32.74 \\
\cline { 2 - 4 } & & & Total & 635.00
\end{tabular}

Total biomass in metric tons $=63.50$ 
Appendix 2. Plant Nutrient Composition 
Table 8. Percent of carbon (C) in selected species collected during survey of Shingobee and Williams Lakes

[If more than one sample, the percent of $\mathrm{C}$ is calculated as the mean; No. = number; $\%=$ percent; Std $=$ standard deviation]

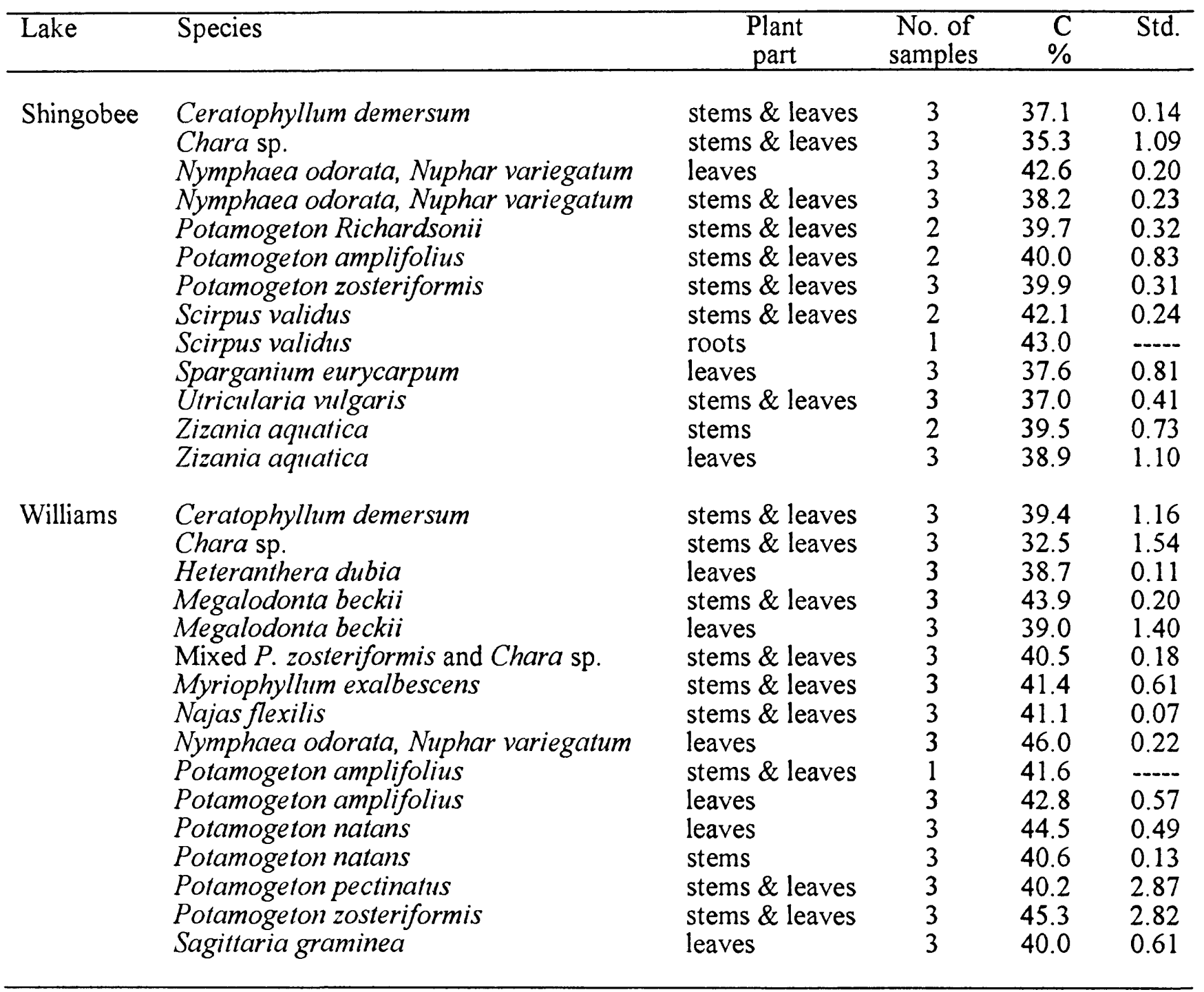


Table 9. Percent of nitrogen $(\mathrm{N})$ in selected species collected during survey of Shingobee and Williams Lakes

[If more than one sample, the percent of $\mathrm{N}$ is calculated as the mean; No. = number; $\%=$ percent; Std $=$ standard deviation $]$

\begin{tabular}{|c|c|c|c|c|c|}
\hline Lake & species & $\begin{array}{l}\text { plant } \\
\text { part }\end{array}$ & $\begin{array}{c}\text { No. of } \\
\text { samples }\end{array}$ & $\begin{array}{l}\mathbf{N} \\
\%\end{array}$ & Std \\
\hline \multirow[t]{13}{*}{ Shingobee } & Ceratophyllum demersum & stems \& leaves & 3 & 2.0 & 0.04 \\
\hline & Chara sp. & stems \& leaves & 3 & 2.3 & 0.29 \\
\hline & Nymphaea odorata, Nuphar variegatum & leaves & 3 & 2.9 & 0.41 \\
\hline & Nymphaea odorata, Nuphar variegatum & stems \& leaves & 3 & 1.4 & 0.30 \\
\hline & Potamogeton Richardsonii & stems \& leaves & 2 & 2.4 & 0.06 \\
\hline & Potamogeton amplifolius & stems \& leaves & 2 & 2.2 & 0.57 \\
\hline & Potamogeton zosteriformis & stems \& leaves & 3 & 2.0 & 0.17 \\
\hline & Scirpus validus & stems \& leaves & 2 & 0.7 & 0.14 \\
\hline & Scirpus validus & roots & 1 & 0.5 & \\
\hline & Sparganium eurycarpum & leaves & 3 & 1.4 & 0.37 \\
\hline & Utricularia vulgaris & stems \& leaves & 3 & 2.0 & 0.04 \\
\hline & Zizania aquatica & stems & 2 & 1.1 & 0.07 \\
\hline & Zizania aquatica & leaves & 3 & 2.4 & 0.10 \\
\hline \multirow[t]{16}{*}{ Williams } & Ceratophyllum demersum & stems \& leaves & 3 & 2.2 & 0.12 \\
\hline & Chara sp. & stems \& leaves & 3 & 2.1 & 0.11 \\
\hline & Heteranthera dubia & leaves & 3 & 1.7 & 0.10 \\
\hline & Megalodonta beckii & stems \& leaves & 3 & 1.8 & 0.11 \\
\hline & Megalodonta beckii & leaves & 3 & 2.6 & 0.31 \\
\hline & Mixed P. zosteriformis and Chara sp. & stems \& leaves & 3 & 1.7 & 0.04 \\
\hline & Myriophyllum exalbescens & stems \& leaves & 3 & 2.1 & 0.13 \\
\hline & Najas flexilis & stems \& leaves & 3 & 1.8 & 0.11 \\
\hline & Nymphaea odorata, Nuphar variegatum & leaves & 3 & 2.9 & 0.17 \\
\hline & Potamogeton amplifolius & stems \& leaves & 1 & 1.4 & \\
\hline & Potamogeton amplifolius & leaves & 3 & 1.9 & 0.23 \\
\hline & Potamogeton natans & leaves & 3 & 2.8 & 0.23 \\
\hline & Potamogeton natans & stems & 3 & 1.3 & 0.02 \\
\hline & Potamogeton pectinatus & stems \& leaves & 3 & 1.7 & 0.30 \\
\hline & Potamogeton zosteriformis & stems \& leaves & 3 & 2.4 & 0.09 \\
\hline & Sagittaria graminea & leaves & 3 & 2.2 & 0.01 \\
\hline
\end{tabular}


Table 10. Percent of hydrogen $(\mathrm{H})$ in selected species collected during survey of Shingobee and Williams Lakes

[If more than one sample, the percent of $\mathrm{H}$ is calculated as the mean; No. = number; $\%=$ percent; Std $=$ standard deviation $]$

\begin{tabular}{|c|c|c|c|c|c|}
\hline Lake & species & $\begin{array}{l}\text { plant } \\
\text { part }\end{array}$ & $\begin{array}{c}\text { No. of } \\
\text { samples }\end{array}$ & $\begin{array}{l}\mathrm{H} \\
\% \\
\end{array}$ & Std \\
\hline \multirow[t]{13}{*}{ Shingobee } & Ceratophyllum demersum & stems \& leaves & 3 & 5.7 & 0.06 \\
\hline & Chara sp. & stems \& leaves & 3 & 5.8 & 0.26 \\
\hline & Nymphaea odorata, Nuphar variegatum & leaves & 3 & 5.8 & 0.16 \\
\hline & Nymphaea odorata, Nuphar variegatum & stems \& leaves & 3 & 5.6 & 0.06 \\
\hline & Potamogeton Richardsonii & stems \& leaves & 2 & 6.1 & 0.11 \\
\hline & Potamogeton amplifolius & stems \& leaves & 2 & 6.1 & 0.23 \\
\hline & Potamogeton zosteriformis & stems \& leaves & 3 & 6.2 & 0.06 \\
\hline & Scirpus validus & stems \& leaves & 2 & 6.0 & 0.06 \\
\hline & Scirpus validus & roots & 1 & 6.1 & $-\ldots$ \\
\hline & Sparganium eurycarpum & leaves & 3 & 5.5 & 0.13 \\
\hline & Utricularia vulgaris & stems \& leaves & 3 & 5.7 & 0.01 \\
\hline & Zizania aquatica & stems & 2 & 5.6 & 0.09 \\
\hline & Zizania aquatica & leaves & 3 & 5.6 & 0.16 \\
\hline \multirow[t]{16}{*}{ Williams } & Ceratophyllum demersum & stems \& leaves & 3 & 6.1 & 0.08 \\
\hline & Chara sp. & stems \& leaves & 3 & 4.7 & 0.29 \\
\hline & Heteranthera dubia & leaves & 3 & 5.6 & 0.04 \\
\hline & Megalodonta beckii & stems \& leaves & 3 & 6.3 & 0.11 \\
\hline & Megalodonta beckii & leaves & 3 & 5.6 & 0.25 \\
\hline & Mixed $P$. zosteriformis and Chara sp. & stems \& leaves & 3 & 5.8 & 0.01 \\
\hline & Myriophyllum exalbescens & stems \& leaves & 3 & 6.1 & 0.08 \\
\hline & Najas flexilis & stems \& leaves & 3 & 6.4 & 0.17 \\
\hline & Nymphaea odorata, Nuphar variegatum & leaves & 3 & 6.2 & 0.02 \\
\hline & Potamogeton amplifolius & stems \& leaves & 1 & 6.5 & \\
\hline & Potamogeton amplifolius & leaves & 3 & 6.2 & 0.06 \\
\hline & Potamogeton natans & leaves & 3 & 6.3 & 0.03 \\
\hline & Potamogeton natans & stems & 3 & 6.1 & 0.09 \\
\hline & Potamogeton pectinatus & stems \& leaves & 3 & 5.9 & 0.32 \\
\hline & Potamogeton zosteriformis & stems \& leaves & 3 & 7.0 & 0.43 \\
\hline & Sagittaria graminea & leaves & 3 & 6.1 & 0.10 \\
\hline
\end{tabular}


Table 11. Percent of phosphorus (P) in selected species collected during survey of Shingobee and Williams Lakes

[If more than one sample, the percent of $\mathrm{P}$ is calculated as the mean; No. $=$ number; $\%=$ percent; Std $=$ standard deviation $]$

\begin{tabular}{|c|c|c|c|c|c|}
\hline Lake & species & $\begin{array}{l}\text { plant } \\
\text { part }\end{array}$ & $\begin{array}{c}\text { No. of } \\
\text { samples }\end{array}$ & $\begin{array}{r}\mathrm{P} \\
\% \\
\end{array}$ & Std \\
\hline \multirow[t]{13}{*}{ Shingobee } & Ceratophyllum demersum & stems \& leaves & 3 & 0.2 & 0.01 \\
\hline & Chara sp. & stems \& leaves & 3 & 0.1 & 0.02 \\
\hline & Nymphaea odorata, Nuphar variegatum & leaves & 3 & 0.2 & 0.05 \\
\hline & Nymphaea odorata, Nuphar variegatum & stems \& leaves & 3 & 0.2 & 0.06 \\
\hline & Potamogeton Richardsonii & stems \& leaves & 2 & 0.2 & 0.01 \\
\hline & Potamogeton amplifolius & stems \& leaves & 2 & 0.2 & 0.04 \\
\hline & Potamogeton zosteriformis & stems \& leaves & 3 & 0.1 & 0.03 \\
\hline & Scirpus validus & stems \& leaves & 2 & 0.1 & 0.01 \\
\hline & Scirpus validus & roots & 1 & 0.1 & $-\cdots$ \\
\hline & Sparganium eurycarpum & leaves & 3 & 0.2 & 0.06 \\
\hline & Utricularia vulgaris & stems \& leaves & 3 & 0.1 & 0.02 \\
\hline & Zizania aquatica & stems & 2 & 0.2 & 0.02 \\
\hline & Zizania aquatica & leaves & 3 & 0.1 & 0.08 \\
\hline \multirow[t]{16}{*}{ Williams } & Ceratophyllum demersum & stems \& leaves & 3 & 0.1 & 0.01 \\
\hline & Chara sp. & stems \& leaves & 3 & 0.1 & 0.00 \\
\hline & Heteranthera dubia & leaves & 3 & 0.1 & 0.01 \\
\hline & Megalodonta beckii & stems \& leaves & 3 & 0.1 & 0.00 \\
\hline & Megalodonta beckii & leaves & 3 & 0.1 & 0.01 \\
\hline & Mixed P. zosteriformis and Chara sp. & stems \& leaves & 3 & 0.1 & 0.00 \\
\hline & Myriophyllum exalbescens & stems \& leaves & 3 & 0.1 & 0.01 \\
\hline & Najas flexilis & stems \& leaves & 3 & 0.1 & 0.02 \\
\hline & Nymphaea odorata, Nuphar variegatum & leaves & 3 & 0.1 & 0.02 \\
\hline & Potamogeton amplifolius & stems \& leaves & 1 & 0.1 & ---- \\
\hline & Potamogeton amplifolius & leaves & 3 & 0.1 & 0.02 \\
\hline & Potamogeton natans & leaves & 3 & 0.1 & 0.00 \\
\hline & Potamogeton natans & stems & 3 & 0.1 & 0.01 \\
\hline & Potamogeton pectinatus & stems \& leaves & 3 & 0.1 & 0.01 \\
\hline & Potamogeton zosteriformis & stems \& leaves & 3 & 0.1 & 0.00 \\
\hline & Sagittaria graminea & leaves & 3 & 0.1 & 0.01 \\
\hline
\end{tabular}


Table 12. Percent of potassium (K) in selected species collected during survey of Shingobee and Williams Lakes

[If more than one sample, the percent of $\mathrm{K}$ is calculated as the mean; No. = number; $\%=$ percent; Std $=$ standard deviation]

\begin{tabular}{|c|c|c|c|c|c|}
\hline Lake & species & $\begin{array}{l}\text { plant } \\
\text { part }\end{array}$ & $\begin{array}{c}\text { No. of } \\
\text { samples }\end{array}$ & $\begin{array}{l}\mathrm{K} \\
\% \\
\end{array}$ & $\overline{\text { Std }}$ \\
\hline \multirow[t]{13}{*}{ Shingobee } & Ceratophyllum demersum & stems \& leaves & 3 & 0.1 & 0.00 \\
\hline & Chara sp. & stems \& leaves & 3 & 0.1 & 0.05 \\
\hline & Nymphaea odorata, Nuphar variegatum & leaves & 3 & 1.2 & 0.29 \\
\hline & Nymphaea odorata, Nuphar variegatum & stems \& leaves & 3 & 2.3 & 0.11 \\
\hline & Potamogeton Richardsonii & stems \& leaves & 2 & 0.3 & 0.01 \\
\hline & Potamogeton amplifolius & stems \& leaves & 2 & 0.2 & 0.00 \\
\hline & Potamogeton zosteriformis & stems \& leaves & 3 & 0.1 & 0.05 \\
\hline & Scirpus validus & stems \& leaves & 2 & 0.8 & 0.04 \\
\hline & Scirpus validus & roots & 1 & 0.8 & ---- \\
\hline & Sparganium eurycarpum & leaves & 3 & 3.1 & 0.61 \\
\hline & Utricularia vulgaris & stems \& leaves & 3 & 0.2 & 0.04 \\
\hline & Zizania aquatica & stems & 2 & 0.9 & 0.05 \\
\hline & Zizania aquatica & leaves & 3 & 0.4 & 0.50 \\
\hline \multirow[t]{16}{*}{ Williams } & Ceratophyllum demersum & stems \& leaves & 3 & 0.1 & 0.00 \\
\hline & Chara sp. & stems \& leaves & 3 & 0.1 & 0.01 \\
\hline & Heteranthera dubia & leaves & 3 & 0.1 & 0.03 \\
\hline & Megalodonta beckii & stems \& leaves & 3 & 0.1 & 0.01 \\
\hline & Megalodonta beckii & leaves & 3 & 0.2 & 0.12 \\
\hline & Mixed $P$. zosteriformis and Chara sp. & stems \& leaves & 3 & 0.9 & 0.03 \\
\hline & Myriophyllum exalbescens & stems \& leaves & 3 & 0.1 & 0.00 \\
\hline & Najas flexilis & stems \& leaves & 3 & 0.1 & 0.00 \\
\hline & Nymphaea odorata, Nuphar variegatum & leaves & 3 & 0.2 & 0.02 \\
\hline & Potamogeton amplifolius & stems \& leaves & 1 & 0.4 & ---- \\
\hline & Potamogeton amplifolius & leaves & 3 & 0.3 & 0.10 \\
\hline & Potamogeton natans & leaves & 3 & 0.0 & 0.01 \\
\hline & Potamogeton natans & stems & 3 & 0.1 & 0.02 \\
\hline & Potamogeton pectinatus & stems \& leaves & 3 & 0.2 & 0.04 \\
\hline & Potamogeton zosteriformis & stems \& leaves & 3 & 0.1 & 0.00 \\
\hline & Sagittaria graminea & leaves & 3 & 0.4 & 0.05 \\
\hline
\end{tabular}


Table 13. Percent of calcium $(\mathrm{Ca})$ in selected species collected during survey of Shingobee and Williams Lakes

[If more than one sample, the percent of $\mathrm{Ca}$ is calculated as the mean; No. $=$ number; $\%=$ percent; Std $=$ standard deviation $]$

\begin{tabular}{|c|c|c|c|c|c|}
\hline Lake & species & $\begin{array}{l}\text { plant } \\
\text { part }\end{array}$ & $\begin{array}{c}\text { No. of } \\
\text { samples }\end{array}$ & $\begin{array}{l}\mathrm{Ca} \\
\% \\
\end{array}$ & Std \\
\hline \multirow[t]{13}{*}{ Shingobee } & Ceratophyllum demersum & stems \& leaves & 3 & 2.4 & 0.01 \\
\hline & Chara sp. & stems \& leaves & 3 & 3.3 & 1.13 \\
\hline & Nymphaea odorata, Nuphar variegatum & leaves & 3 & 2.0 & 1.04 \\
\hline & Nymphaea odorata, Nuphar variegatum & stems \& leaves & 3 & 1.4 & 0.25 \\
\hline & Potamogeton Richardsonii & stems \& leaves & 2 & 1.6 & 0.10 \\
\hline & Potamogeton amplifolius & stems \& leaves & 2 & 1.9 & 0.34 \\
\hline & Potamogeton zosteriformis & stems \& leaves & 3 & 1.5 & 0.32 \\
\hline & Scirpus validus & stems \& leaves & 2 & 0.2 & 0.00 \\
\hline & Scirpus validus & roots & 1 & 0.2 & --.- \\
\hline & Sparganium eurycarpum & leaves & 3 & 2.4 & 0.60 \\
\hline & Utricularia vulgaris & stems \& leaves & 3 & 2.3 & 0.21 \\
\hline & Zizania aquatica & stems & 2 & 0.4 & 0.04 \\
\hline & Zizania aquatica & leaves & 3 & 0.6 & 0.82 \\
\hline \multirow[t]{16}{*}{ Williams } & Ceratophyllum demersum & stems \& leaves & 3 & 4.1 & 0.68 \\
\hline & Chara sp. & stems \& leaves & 3 & 11.4 & 1.03 \\
\hline & Heteranthera dubia & leaves & 3 & 3.2 & 0.10 \\
\hline & Megalodonta beckii & stems \& leaves & 3 & 1.7 & 0.07 \\
\hline & Megalodonta beckii & leaves & 3 & 4.4 & 1.71 \\
\hline & Mixed $P$. zosteriformis and Chara sp. & stems \& leaves & 3 & 2.1 & 0.17 \\
\hline & Myriophyllum exalbescens & stems \& leaves & 3 & 2.2 & 0.51 \\
\hline & Najas flexilis & stems \& leaves & 3 & 2.3 & 0.30 \\
\hline & Nymphaea odorata, Nuphar variegatum & leaves & 3 & 1.5 & 0.02 \\
\hline & Potamogeton amplifolius & stems \& leaves & 1 & 2.0 & \\
\hline & Potamogeton amplifolius & leaves & 3 & 2.3 & 0.06 \\
\hline & Potamogeton natans & leaves & 3 & 1.7 & 0.17 \\
\hline & Potamogeton natans & stems & 3 & 2.5 & 0.08 \\
\hline & Potamogeton pectinatus & stems \& leaves & 3 & 3.0 & 1.08 \\
\hline & Potamogeton zosteriformis & stems \& leaves & 3 & 1.8 & 0.18 \\
\hline & Sagittaria graminea & leaves & 3 & 2.2 & 0.38 \\
\hline
\end{tabular}


Table 14. Percent of magnesium $(\mathrm{Mg})$ in selected species collected during survey of Shingobee and Williams Lakes

[If more than one sample, the percent of $\mathrm{Mg}$ is calculated as the mean; No. = number; $\%=$ percent; Std $=$ standard deviation $]$

\begin{tabular}{|c|c|c|c|c|c|}
\hline Lake & species & $\begin{array}{l}\text { plant } \\
\text { part }\end{array}$ & $\begin{array}{c}\text { No. of } \\
\text { samples }\end{array}$ & $\begin{array}{r}\mathrm{Mg} \\
\%\end{array}$ & $\overline{\text { Std }}$ \\
\hline \multirow[t]{13}{*}{ Shingobee } & Ceratophyllum demersum & stems \& leaves & 3 & 0.4 & 0.06 \\
\hline & Chara sp. & stems \& leaves & 3 & 0.1 & 0.03 \\
\hline & Nymphaea odorata, Nuphar variegatum & leaves & 3 & 0.3 & 0.06 \\
\hline & Nymphaea odorata, Nuphar variegatum & stems \& leaves & 3 & 0.3 & 0.04 \\
\hline & Potamogeton Richardsonii & stems \& leaves & 2 & 0.2 & 0.00 \\
\hline & Potamogeton amplifolius & stems \& leaves & 2 & 0.2 & 0.01 \\
\hline & Potamogeton zosteriformis & stems \& leaves & 3 & 0.2 & 0.02 \\
\hline & Scirpus validus & stems \& leaves & 2 & 0.1 & 0.01 \\
\hline & Scirpus validus & roots & 1 & 0.1 & ----- \\
\hline & Sparganium eurycarpum & leaves & 3 & 0.6 & 0.06 \\
\hline & Utricularia vulgaris & stems \& leaves & 3 & 0.8 & 0.00 \\
\hline & Zizania aquatica & stems & 2 & 0.2 & 0.00 \\
\hline & Zizania aquatica & leaves & 3 & 0.1 & 0.16 \\
\hline \multirow[t]{16}{*}{ Williams } & Ceratophyllum demersum & stems \& leaves & 3 & 0.2 & 0.02 \\
\hline & Chara sp. & stems \& leaves & 3 & 0.4 & 0.06 \\
\hline & Heteranthera dubia & leaves & 3 & 0.2 & 0.00 \\
\hline & Megalodonta beckii & stems \& leaves & 3 & 0.1 & 0.01 \\
\hline & Megalodonta beckii & leaves & 3 & 0.2 & 0.05 \\
\hline & Mixed $P$. zosteriformis and Chara sp. & stems \& leaves & 3 & 0.2 & 0.00 \\
\hline & Myriophyllum exalbescens & stems \& leaves & 3 & 0.1 & 0.01 \\
\hline & Najas flexilis & stems \& leaves & 3 & 0.2 & 0.02 \\
\hline & Nymphaea odorata, Nuphar variegatum & leaves & 3 & 0.2 & 0.01 \\
\hline & Potamogeton amplifolius & stems \& leaves & 1 & 0.2 & --- \\
\hline & Potamogeton amplifolius & leaves & 3 & 0.2 & 0.02 \\
\hline & Potamogeton natans & leaves & 3 & 0.1 & 0.01 \\
\hline & Potamogeton natans & stems & 3 & 0.1 & 0.01 \\
\hline & Potamogeton pectinatus & stems \& leaves & 3 & 0.1 & 0.02 \\
\hline & Potamogeton zosteriformis & stems \& leaves & 3 & 0.1 & 0.01 \\
\hline & Sagittaria graminea & leaves & 3 & 0.3 & 0.03 \\
\hline
\end{tabular}


Appendix 3. Plant Species in the Emergent Zones of Shingobee and Williams Lakes 
Table 15. Plant species identified in the emergent zone of Shingobee Lake.

[The emergent zone was widest along the south and east shores, and narrow along the north and west shores. Soils were primarily organic. The emergent zone was dominated by Carex sp. and Phalaris arundinacea with Phragmites communis and Typha latifolia dominant in some locales. In this table, the emergent zone does not include the floating-leafed zone.]

\begin{tabular}{ll}
\hline Species & Common name \\
\hline & \\
Acer rubrum L. & red maple \\
Agrostis gigantea Roth & black bent \\
Aster sp. & aster \\
Bidens cernua L. & stick-tight \\
Bromus ciliatus L. & brome grass \\
Calamagrostis canadensis (Michx.) Nutt. & blue-joint \\
Caltha palustris L. & cowslip \\
Campanula aparinoides Pursh. & marsh bellflower \\
Carex pseudo-cyperus L. & cyperus-like sedge \\
Carex lanuginosa Michx. & wooly sedge \\
Carex rostrata Stokes & beaked sedge \\
Carex stricta Lam. & tussock sedge \\
Carex trichocarpa Muhl. & sedge \\
Cicuta bulbifera L. & bulb-bearing water-hemlock \\
Cornus stolonifera Michx. & red osier \\
Epilobium leptophyllum Raf. & narrow-leafed willowherb \\
Equisetum sp. & horsetail, scouring-rush \\
Eupatorium sp. & \\
Galium sp. & bedstraw \\
Hippuris vulgaris L. & mare's-tail \\
Impatiens capensis Meerb. & jewelweed \\
Iris virginica & iris \\
Muhlenbergia glomerata (Willd.) Trin. & satin-grass, dropseed \\
Phalaris arundinacea L. & reed canary-grass \\
Phragmites communis Trin. & common reed-grass \\
Picea mariana (Mill.) BSP & black spruce \\
Polygonum lapathifolium L. & smartweed \\
Rumex sp. & dock \\
Sagittaria latifolia Willd. & duck-potato \\
Salix sp. & willow \\
Scirpus validus Vahl. & soft-stem bulrush \\
Sparganium androcladum (Engelm.) Morong & branching bur-reed \\
Sparganium eurycarpum Engelm. & broad-fruited bur-reed \\
Typha latifolia L. & common cattail \\
Almus sp. & alder \\
Zizania aquatica L. & wild rice \\
\hline & \\
\hline &
\end{tabular}


Table 16. Plant species identified in the emergent zone of Williams Lake.

[The emergent zone of Williams Lake is narrow and has soils ranging from sands and gravels to organic. The species composition listed below reflects the heterogenicity of the shore line; different species were dominant in different locales. In this table, the emergent zone does not include the floating-leafed zone.]

\begin{tabular}{|c|c|}
\hline Species & Common name \\
\hline Agrostis hyemalis (Walt.) BSP & ticklegrass, hairgrass \\
\hline Bidens cernua $\mathrm{L}$. & stick-tight \\
\hline Calamagrostis canadensis (Michaux) Nutt. & blue joint \\
\hline Calamagrostis neglecta (Ehrh.) Gaertn., Mey. \& Scherb. & reed bentgrass \\
\hline Campanula aparinoides Pursh & marsh bellflower \\
\hline Carex atherodes Spreng. & awned sedge \\
\hline Carex Bebbii Olney & Bebb's sedge \\
\hline Carex hirta $\mathrm{L}$. & hairy sedge \\
\hline Carex hyalinolepis Steud. & sedge \\
\hline Carex hystricina Muhl. & porcupine sedge \\
\hline Carex lasiocarpa Ehrh. & slender sedge \\
\hline Carex rostrata Stokes & beaked sedge \\
\hline Carex viridula Michx. & sedge \\
\hline Carex vulpinoidea Michx. & sedge \\
\hline Cicuta bulbifera $\mathrm{L}$. & bulb-bearing water hemlock \\
\hline Echinochloa muricata (Beauv.) Fern. & grass \\
\hline Eleocharis acicularis (L.) R.\& S. & needle spike-rush \\
\hline Eleocharis Smallii Britt. & Small's spike-rush \\
\hline Epilobium glandulosum Lehm. & willow-herb \\
\hline Epilobium leptophyllum Raf. & narrow-leaved willow-herb \\
\hline Equisetum sp. & horsetail \\
\hline Fraxinus pennsylvanica Marsh. & red ash \\
\hline Glyceria borealis (Nash) Batchelder & float-grass \\
\hline Glyceria grandis S.Wats. & reed-meadow grass \\
\hline Glyceria striata (Lam.) Hitchc. & fowl-meadow grass \\
\hline Glyceria striata var stricta (Scribn.) Fern. & fowl-meadow grass \\
\hline Hypericum punctatum Lam. & spotted St. John's-wort \\
\hline Impatiens capensis Meers. & jewelweed \\
\hline Juncus brevicaudatus(Engelm.) Fern. & narrow-panicled rush \\
\hline Juncus Dudleyi Wieg. & Dudley's rush \\
\hline Juncus nodosus L. & knotted rush \\
\hline Linaria canadensis (L.) Dumont & old-field-toadflax \\
\hline Lobelia siphilicita $\mathrm{L}$. & blue cardinal flower \\
\hline Lycopus americanus Muhl. & water-horehound \\
\hline Muhlenbergia mexicana (L.) Trin. & satin-grass \\
\hline Phalaris arundinacea $\mathrm{L}$. & reed-canary-grass \\
\hline
\end{tabular}


Table 16. Plant species identified in the emergent zone of Williams Lake -continued

[The emergent zone of Williams Lake is narrow and has soils ranging from sands and gravels to organic. The species composition listed below reflects the heterogenicity of the shore line; different species were dominant in different locales. In this table, the emergent zone does not include the floating-leafed zone.]

\begin{tabular}{ll}
\hline Species & Common name \\
\hline & \\
Poa compressa L. & Canada bluegrass, wiregrass \\
Poa palustris L. & fowl-meadowgrass \\
Polygonum lapathifolium L. & smartweed \\
Sagittaria graminea Michx. & grass-leafed arrowhead \\
Sagittaria latifolia f. gracilis (Pursh) Robins. & arrowhead \\
Sagittaria latifolia Willd. & duck-potato \\
Salix interior Rowlee & sandbar-willow \\
Salix sp. & willow \\
Scirpus atrovirens Willd. & dark-green bulrush \\
Scirpus cyperinus (L.) Kunth & wool-grass \\
Scirpus Smithii Gray & bulrush \\
Scirpus validus Vahl. & soft-stem bulrush \\
Setaria glauca (L.) Beauv. & foxtail, pigeon-grass \\
Typha latifolia L. & common cattail \\
Utricularia subulata L. & bladderwort \\
\hline
\end{tabular}


Appendix 4. Lake Hypsographic Data 
Table 17. Hypsographic and depth-volume data for Williams Lake

$$
\text { [m = meters }]
$$

\begin{tabular}{ccccc}
\hline Depth $(\mathrm{m})$ & $\begin{array}{c}\text { Area } \\
\mathrm{m}^{2} \times 10^{5}\end{array}$ & Stratum $(\mathrm{m})$ & $\begin{array}{c}\text { Volume } \\
\mathrm{m}^{3} \times 10^{5}\end{array}$ & $\begin{array}{c}\text { Percent of } \\
\text { total volume }\end{array}$ \\
\hline 0 & 3.946 & $0-1$ & 3.757 & 18.5 \\
1 & 3.571 & $1-2$ & 3.299 & 16.3 \\
2 & 3.034 & $2-3$ & 2.816 & 13.9 \\
3 & 2.602 & $3-4$ & 2.473 & 12.2 \\
4 & 2.346 & $4-5$ & 2.215 & 10.9 \\
5 & 2.086 & $5-6$ & 1.962 & 9.7 \\
6 & 1.841 & $6-7$ & 1.640 & 8.1 \\
7 & 1.446 & $7-8$ & 1.229 & 6.1 \\
8 & 1.024 & $8-9$ & 0.752 & 3.7 \\
9 & 0.510 & $9-9.8$ & 0.136 & 0.7 \\
\hline & & Total $=$ & 20.278 & 100.0
\end{tabular}

Table 18. Hypsographic and depth-volume data for Shingobee Lake

$$
\text { [ } \mathrm{m}=\text { meters] }
$$

\begin{tabular}{ccccc}
\hline Depth $(\mathrm{m})$ & $\begin{array}{c}\text { Area } \\
\mathrm{m}^{2} \times 10^{5}\end{array}$ & $\begin{array}{c}\text { Volume } \\
\text { Stratum }(\mathrm{m})\end{array}$ & $\begin{array}{c}\text { Percent of } \\
\mathrm{m}^{3} \times 10^{5}\end{array}$ & $\begin{array}{c}\text { total volume } \\
\text { talu }\end{array}$ \\
\hline 0 & 6.244 & $0-1$ & 5.954 & 15.6 \\
1 & 5.669 & $1-2$ & 5.458 & 14.3 \\
2 & 5.250 & $2-3$ & 5.126 & 13.5 \\
3 & 5.004 & $3-4$ & 4.852 & 12.8 \\
4 & 4.702 & $4-5$ & 4.453 & 11.7 \\
5 & 4.210 & $5-6$ & 3.833 & 10.1 \\
6 & 3.468 & $6-7$ & 3.054 & 8.0 \\
7 & 2.658 & $7-8$ & 2.284 & 6.0 \\
8 & 1.930 & $8-9$ & 1.629 & 4.3 \\
9 & 1.346 & $9-10$ & 0.986 & 2.6 \\
10 & 0.665 & $10-11$ & 0.373 & 1.0 \\
11 & 0.145 & $11-11.9$ & 0.043 & 0.1 \\
\hline & & Total $=$ & 38.046 & 100.0
\end{tabular}


Appendix 4. Light Attenuation Data 
Table 19. Attenuation coefficients $\left(\mathrm{K}_{\mathrm{PAR}}\right)$ calculated from radiometer data collected in Williams and Shingobee Lakes in 1991 and Secchi depth readings made in 1990

$$
[\mathrm{m}=\text { meters }]
$$

\begin{tabular}{lrrr}
\hline Lake & \multicolumn{2}{c}{ Attenuation coefficients } & $\begin{array}{c}\text { Secchi depth } \\
\text { readings }\end{array}$ \\
\cline { 2 - 4 } Shingobee & Below surface to 2 meters & Below surface to 4 meters & \\
Williams & $0.52 \mathrm{~m}^{-1}$ & $0.57 \mathrm{~m}^{-1}$ & 4 meters \\
& $0.51 \mathrm{~m}^{-1}$ & $0.48 \mathrm{~m}^{-1}$ & 5 meters \\
\hline
\end{tabular}

